Alma Mater Studiorum - Università di Bologna DEPARTMENT OF ECONOMICS

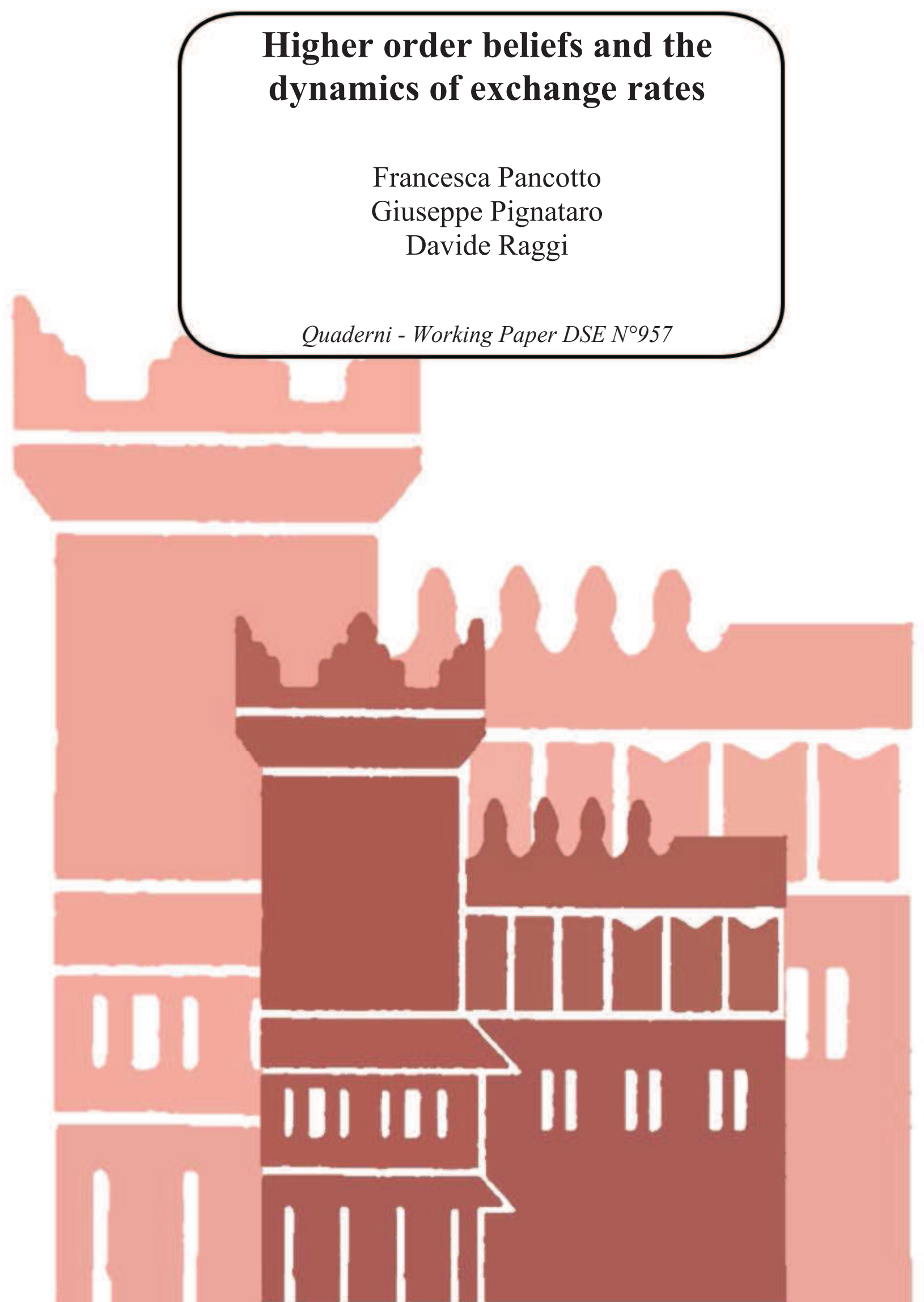




\title{
Higher order beliefs and the dynamics of exchange rates
}

\author{
Francesca Pancotto \\ University of Modena and Reggio Emilia \\ Giuseppe Pignataro \\ University of Bologna \\ Davide Raggi \\ University of Bologna
}

\begin{abstract}
We estimate a model for exchange rate dynamics when expectations present higher order beliefs. A structural macro model for exchange rates is proposed where agents form their one-step-ahead predictions under a Bayesian learning process and in which aggregation of their choices is considered into the dynamics of exchange rates. Bayesian estimation of the structural parameters is implemented by using survey data on heterogeneous forecasts and fundamentals. Results show that higher order beliefs are relevant in building the subjective expectations' process and this phenomenon is dependent on information uncertainty. First, public information coordinates heterogeneous expectations of predictors leading to overweighting of signals from specific fundamentals. Second, predictors mis-perceive them as the most prevalent factors to form their forecasts, although the role of their own private assessments fades away. Keywords: beauty contest, higher order beliefs, exchange rates, economic fundamentals, survey data
\end{abstract}

\footnotetext{
${ }^{\text {th }}$ Financial support from Einaudi Institute of Economics and Finance (EIEF 2011) is gratefully acknowledged. We are grateful to Chris Wallace, Efrem Castelnuovo, Pietro Dindo, as well as to the participants at presentations Bomopav (University of Venice) for their helpful feedback. We wish to thank Tommaso Proietti for providing us with the Ox code for disaggregating quarterly time series. All remaining errors are ours.

Email addresses: francesca.pancotto@gmail.com (Francesca Pancotto), giuseppe.pignataro@unibo.it (Giuseppe Pignataro), davide.raggi@unibo.it (Davide Raggi)
} 


\section{Introduction}

Professional investors have specialized expertise in forecasting exchange rates. For instance, whether you are a business or a trader, having an exchange rate forecast to guide your decision making can be very important to minimize risks and maximize returns. Forecasting exchange rates is a rather difficult task, especially for short run horizons (Meese and Rogoff, 1983). Recent studies based on heterogeneous expectations appear to be a viable strategy to improve predictive abilities of exchange rates (Bacchetta and Van Wincoop, 2006, 2013).

According to a structural model, our interest is to mimic the real process in the market where currency forecasts are produced. In particular, we believe that one of the interesting points in explaining market fluctuations is the role investors play in the exchange rate market. This paper therefore examines how investors' choices should be optimally designed on the basis of the relevant information in place. In our model, the selection process first generates an informative private signal about exchange rates fluctuations. This signal may be depicted as the investor's ability to correctly interpret the real value of the fundamental and it is simply based on the personal and subjective evaluation including opinions, rumors, economic projections and market commentary. Moreover, investors observe a public signal, as sunspot, switching their actions contingent on it. Such sunspot explanations are apparently intended as proxies for the true higher order beliefs explanations. The optimal design in this environment thus depends on the agent's beliefs about economic fundamental, but also on other agents' beliefs.

We model investors' heterogeneity, and the interaction among them, nesting a micro-based learning process into a standard macroeconomic framework with two countries. Each agent forms her expectation by combining both public and private information. The aggregation of all agents' choices is further considered in the recursive dynamics of exchange rates at macro level. In the spirit of Morris and Shin (2002), we first extend the learning process to a dynamic setup. This generalization allows to define a structural time series model that closely mimics our theoretical insights. As a second step, we quantify what is the relevant information investors use in taking their decisions and disentangle the effects of both public and private signals. We also identify the weights that the information has in determining agents' choices. Our third contribution is to evaluate the structural parameters of the model cast in a statespace form using Bayesian techniques. Inference on expectations is based on a panel of survey data whose relevant feature is to be heterogeneous among agents as well as on standard macroeconomic fundamentals.

In particular, our analysis builds on the micro-structure of exchange rate predictions with dispersed 
information on fundamentals, while we test its relevance on the aggregate macro-dynamics of the eur/usd currency for the period ranging from 2006 to 2012. The information that traders optimally choose to collect is determined by their intent to correctly codify changes in fundamentals as well as by their interest to align their predictions with those of the others. A beauty contest framework in analogy to Keynes (1936) is thus introduced as agents take into account other agent's expectations when forming their own. ${ }^{1}$ A dynamic game of incomplete information is thus modeled to evaluate the role of higher order beliefs in exchange rate predictions. In particular, a social learning process interchanges the amount of private and public information effectively used by agents and relates the discrepancy between the two sources to the role that higher order beliefs play in determining outcomes. We search whether a potential nexus exists among the choices of market predictors. Then we identify the precise link guiding this micro-strategic behavior to the aggregate macro dynamics. The choice of agents in the currency is consequently tested on the basis of one-period ahead survey predictions provided by heterogeneous professional forecasters. The estimation of structural parameters provides two main findings. First, individual predictions about future values of the currency rely heavily on the role of higher order beliefs. We find that the value measuring higher order beliefs in the decision process of predictors accounts for about 82 per cent. Secondly, public information plays the most important role in determining individual forecasts, i.e., more than 75 per cent, compared to private information. A fairly robust insight from our analysis show that, when forming predictions, investors are heavily biased towards public information instead of focusing on their own personal assessment. Information uncertainty and heterogeneity lead to overweighting signals of the fundamental partially neglecting personal evaluation.

The remainder of this paper is as follows: Section 2 encompasses the relevant literature in the field, while, section 3 introduces the theoretical set-up and equilibrium solutions both at micro and macro levels. Section 4 presents the empirical estimation of the structural model, while some comments are proposed on Section 5 about the posterior estimates and policy implications. Section 6 concludes.

\footnotetext{
${ }^{1}$ Throughout the paper, we use the terms 'beauty contest' or 'higher order beliefs' interchangeably. The term beauty contest is due to Keynes' famous claim that to form their demand for an asset, investors behave as individuals in a beauty contest where people were asked to guess not the prettiest girl among those presented in the newspaper contest, but the girl they thought that the majority would consider the prettiest. He suggests an analogy to financial markets claiming that agents do not only try to generate forecasts to predict the future behavior of assets but also try to guess other market participants' forecasts and the forecasts of forecasts of the others.
} 


\section{Literature review}

Modelling the behaviour of exchange rate has been one of the most challenging tasks demanding the attention of economists in the last thirty years. Meese and Rogoff $(1983)$ were the first to point out the impossibility of structural and time series exchange rate models to outperform a random walk in terms of forecasting ability. This discouraging result seemed partially overcome at least for long run predictions (1 to 3 years forecasts) with the use of cointegration analysis $2^{2}$ Further the application of Taylor rule basedfundamental $\mathrm{s}^{3}$ and of the informational value for order flow ${ }^{4}$ were considered the only possible solutions to improve the quality of exchange rate forecasts over short horizons. Indeed forecasting methodology has obtained better performance after the introduction of the micro-based structure in modelling exchange rates. Several studies underline the role that the interactions among investors may have in explaining the effective dynamics of exchange rates. This is possible by accounting for agents' heterogeneity and their evaluation on public and private information 5 For instance, O'Hara (1995) stressed how the regulation of trading in exchange markets has important implications for the process of price formation and more generally for all unobservable relevant characteristics. Furthermore, the utility-based new open economy macroeconomic framework by Devereux and Engel (2002) or the rational expectations present value model by Engel and West (2005) were proposed as different alternatives motivating the use of heterogeneous information of agents in asset pricing models. More specifically for exchange rates, various contributions of Bacchetta and Van Wincoop (2012) explored in various directions the implications of heterogeneity of expectations in currency markets.

Our paper relates to the whole fast-growing literature on heterogeneity of expectations and higher order beliefs. The most important contributions belong to a series of papers proposed by Bacchetta and van Wincoop who have explored the implication of heterogeneity in expectations on different theoretical

\footnotetext{
$\sqrt[2]{\text { Mark }}(1995)$ and Chinn and Meese $(1995)$ found a substantial improvement in forecasting exchange rates compared to the random walk model, using an error correction term structure over horizons of 2-3 years. These results were nonetheless criticized as time dependent by Faust et al. (2003) and Cheung et al. (2005). A rise in forecasting ability of the monetary models was later ensured by the panel cointegration methods. Husted and MacDonald (1998) used Pedroni (1999) panel cointegration test, while Groen (2005) showed outperformance of a random walk in a panel of three currencies for short and long run. Nonetheless, Mark and Sul (2001) used a panel cointegration version of Mark's paper over 17 currencies with no statistical evidence of outperformance and a smaller forecasting error. Cerra and Saxena (2010) discovered a rise in the predictive success in the long run using a large panel of currencies.

${ }^{3}$ See Molodtsova et al. (2008, 2011) and Molodtsova and Papell (2009).

${ }^{4}$ See Evans and Lyons $(2005)$.

${ }^{5}$ Since the classical contribution of Harsanyi (1967) the rational behavior in such environments depends not only on economic agents' belief about fundamentals, but is also a function of beliefs of higher order, i.e., player's beliefs about other players' beliefs about other players' belief and so on.
} 
models and propose the so-called scapegoat theory of the disconnect between exchange rates and fundamentals. They suppose the existence of uncertainty in the market about the true source of exchange rate fluctuations. When agents observe currency movements that are inconsistent with their expectations, they search for an explanation to these unexpected changes. A weight, higher than average, is assigned to some fundamentals taken as scapegoats. The heterogeneity is modelled looking at investors who receive private signals about the persistence of shocks. They are not able to capture whether the fluctuation on exchange rate is motivated by unobserved factors or simply by a weight, larger than expected one, assigned to macro fundamentals. The latter could be interpreted as a scapegoat, so that the weights attributed to them systematically change over time, thus determining parameter instability. Fratzscher et al. (2012) develop an empirical test of this theory using as a proxy of scapegoat fundamentals, Consensus Economics of London surveys of predictors. The predictors of this panel are asked to rate on a quantitative scale the importance of six key variables (short-term interest rates, long-term interest rates, growth, inflation, current account, equity flows) as drivers of the dynamics of the exchange rate. The authors find that the inclusion of these expectations in the model of exchange rate determination improves the power of the fundamentals in explaining currency movements 6 Bacchetta and Van Wincoop (2006) focus instead on the role of order flow rather than macroeconomic characteristics of the market and introduce a possible explanation for the empirical results verified by Evans and Lyons (2002), Payne (2003), and Froot and Ramadorai (2005).7 Assuming that agents are risk averse with heterogeneous information on the fundamental values, the authors show that due to the imperfect correlated signals among investors, transitory shocks may continuously influence the dynamics of exchange rate. These results originate from a counterbalance effect of risk aversion and uncertainty of information. On one side, the risk-sharing impact is justified since traders must be compensated for the extra risk assumed as a consequence of their actions. On the other side, the uncertainty of information matters since in-

\footnotetext{
${ }^{6}$ In the short run the heterogeneity in the individual evaluation may lead to overrate the random macroeconomic fundamental.

[Evans and Lyons (2002) exploit data pertaining to bilateral transactions among FX dealers via Reuters Dealing 2000-1 electronic trading system. They follow Meese and Rogoff (1983)'s methodology to investigate the out of sample forecasting ability of their linear model. Unfortunately, they do not take into account potential issue of simultaneity bias emerging when exchange rate movements cause order flow. In order to evaluate the possible feed-back effects of exchange rates on order flow, an alternative methodology was suggested by Payne (2003). He elaborates a $V A R$ model estimating information on the size of transactions. This methodology allows for a more precise estimation of the information provided by the order flow. Froot and Ramadorai (2005) extend the framework of Payne (2003) considering inflation and interest rate differentials alongside order flow and excess returns. They also estimate long-run effects of international flows on exchange rates and their relation to fundamentals proposing a decomposition of permanent and transitory components of asset returns.
} 
vestors may confuse an appreciation or depreciation of the exchange rate caused by a liquidity shock with that induced by information on fundamentals. Therefore, if this mix-up concerns information that becomes public in the relative distant future, the impact of order flow on exchange rates is amplified by the infinite regress of investors' individual beliefs. Given the observed prices and quantities, they learn, not only the fundamental values of foreign currencies, but also the other investors' forecasts. The social learning process further disorientates about the informational role that liquidity and fundamental shocks may assume, thus extending the impact of order flow on exchange rates 8 In particular Bacchetta and Van Wincoop (2013) hypothesize a heterogeneous information structure with public and private signals received by forecast market predictors. Each agent needs to keep into account the average expectation of other market participants about the next $t$ - periods exchange rates, when forming her expectation. The difference existing between the expectations about the others' expectations and the actual fundamental, difficult to perceive in the short run, generates the observed wedge between the actual dynamics of the rate and the fundamentals in the long run.

As regards instead the social learning structure of our model, our attention is devoted to a recent literature which tries to understand whether public information improves the effectiveness of policies and is beneficial to markets since it reduces asymmetric information. This framework was mainly popularized by Morris and Shin (2002) and perfectly explains the dynamics of coordination among agents. When coordination incentives are not very strong in the society, higher precision on public information could be in principle welfare-improving. When agents have higher incentives to coordinate, more weight is given to the public signal relative to the private one in the choice of equilibrium actions. Thus it is possible that a potential overreaction to the public signal cancels out the impact of private information. Under certain conditions, this means that a public announcement may destabilize markets, reduce efficiency due to their impacts on higher-order beliefs and can be detrimental for the welfare of agents. This approach has been used as a static representation of many settings with incomplete information and strategic interaction including financial markets (Allen et al., 2006), business cycle models (Angeletos and La'O, 2009, Myatt and Wallace, 2014a), investment decisions (Angeletos et al., 2012; Myatt and Wallace, 2012), price adjustment with monopolistic competition (Myatt and Wallace, 2014b; Woodford, 2002).

Finally for what involves the heterogeneity of survey predictions, there exists an extensive empirical

\footnotetext{
${ }^{8}$ Note that if the private signal involves imminent shifts in fundamentals, this effect is practically thinned down. Intuitively, when private signal concerns next period realizations of fundamentals, there is no need to extract any information from other investors' forecasts since they will share information on fundamentals in a short time.
} 
literature on rationality and inefficiency of predictions. The seminal paper in this field is Ito (1990) which tests individual biases and idiosyncratic effects for a set of disaggregate expectations about the 1-, 3- and 6-month-ahead JY/US rate from the JCIF survey over the period 1985 - 1987 finding substantial heterogeneity among predictors.$^{9}$ Similar results can be confirmed by Elliott and Ito (1999) and Benassy-Quere et al. (2003). MacDonald and Ian (1996) replicate Ito's test for 3- and 12-monthahead estimates of the BP/usd, DM/usd and JY/usd rates from the 1989-1992 and find significant evidence of heterogeneous expectations. Extending the Consensus data set to 1995, Chionis and MacDonald (1997) confirm the presence of individual effects for predictors. Mitchell and Pearce (2007) conducted a thorough analysis of unbiasedness and success rate of predictions along with tests for heterogeneity and strategic forecasting, finding systematic heterogeneity in predictions ${ }^{10}$

\section{Theoretical Model}

The macroeconomic model is a standard two-country monetary model identified by the following basic relationships. Define $E_{t}$ as the nominal exchange rate between home and foreign countries, where $P_{t}$ is the level of home prices and $P_{t}^{*}$ is the level of prices in the foreign country ${ }^{11}$ We assume that Purchasing Power Parity $(P P P)$ holds,

$$
E_{t}=\frac{P_{t}}{P_{t}^{*}}
$$

implying that the exchange rate at which two currencies trade equals the price levels of the two countries.

Rearranging this expression in log terms, we get:

$$
p_{t}=p_{t}^{*}+s_{t}
$$

where $p_{t}$ and $p_{t}^{*}$ are, respectively, the log of home and foreign price level, while $s_{t}$ is the $\log$ of real exchange rate between home and foreign country, i.e., $s_{t}=\log \left(E_{t}\right)$. The second building block of the

\footnotetext{
${ }^{9}$ This literature stems from the recent availability of individual survey-predictions of exchange rates. Previously, a long strand of literature has studied inefficiency and irrationality of exchange rates forecasts. Dominguez (1986) tests the efficiency of foreign exchange market showing that predictors systematically fail in forecasting in the magnitude and the direction of exchange rates movements. Avraham et al. (1987) test the same hypothesis in a high inflationary Israel of the eighties rejecting the notion of rationality of exchange rate expectations. Cavaglia et al. (1993) find that exchange rates forecasts in the EMS are biased. Chinn and Frankel (1994) propose a test rejecting the hypothesis of efficiency and unbiasedness of exchange rate predictions.

${ }^{10}$ For a complete review of the tests about heterogeneity hypothesis using disaggregated survey expectations of professional forecasters, see Jongen et al. (2008).

${ }^{11}$ The starred superscripts usually indicate the variables for foreign country.
} 
model is determined by the money demand for each country, proportional to nominal income 12

The money demand, $M_{t}^{d}$, increases to hold money for transactional purposes and is negatively related to interest rate. Let us denote $L_{t}$ as the negative function of $i_{t}$. It refers to the negative opportunity cost of holding money, instead of an interest-earning asset paying $i$, e.g., $i_{t}=-\log L_{t}$. Long run equilibrium in the money market is achieved when the real money supply, determined by the central bank and indicated by $M_{t}$, is equal to the real demand for money balances, i.e., when $M_{t}=M_{t}^{d}$. We assume that there is no a-priori differences in the structure of the two countries such that $\phi$ and $\alpha$ are equal among them. The money market equilibrium relationship for the two countries at time $t$ is therefore:

$$
\begin{aligned}
\frac{M_{t}}{P_{t}} & =Y_{t}^{\phi} L_{t}^{\alpha}(i) \\
\frac{M_{t}^{*}}{P_{t}^{*}} & =Y_{t}^{* \phi} L_{t}^{\alpha}\left(i^{*}\right)
\end{aligned}
$$

Rewriting both expressions in log terms,

$$
\begin{aligned}
m_{t}-p_{t} & =\phi y_{t}-\alpha i_{t} \\
m_{t}^{*}-p_{t}^{*} & =\phi y_{t}^{*}-\alpha i_{t}^{*}
\end{aligned}
$$

where $y_{t}$ is the log of real output. Finally, the uncovered interest rate parity $(U I P)$ plus deviations hold. Interest rate returns in both countries are equal after controlling for the expected depreciation of home country, namely

$$
\mathbb{E}_{t}\left(s_{t+1}-s_{t}\right)=i_{t}-i_{t}^{*}+\psi_{t}
$$

where $\psi_{t}$ stands exactly for observed deviations from $U I P{ }^{13}$ Since $U I P$ condition is based on rational expectations and risk neutrality, deviations from UIP can be interpreted either as an expectational error or a risk premium associated with liquidity or hedge trade 14 We can rewrite the variables on fundamentals

\footnotetext{
${ }^{12}$ We assume for simplicity the same functional form for the demand of each country:

$$
\frac{M_{t}^{d}}{P_{t}}=Y_{t}^{\phi} L_{t}^{\alpha}(i) ; \quad \frac{M_{t}^{* d}}{P_{t}^{*}}=Y_{t}^{\phi *} L_{t}^{\alpha}\left(i^{*}\right)
$$

${ }^{13}$ The UIP deviation has been tested in the literature by a series of empirical contributions. See James et al. (2012) for a complete review.

${ }^{14}$ This factor is necessary since information heterogeneity generates a deviation from UIP. This hypothesis is different from the standard rational expectations structure where the perfect knowledge of investor's behavior eliminates any deviation from UIP with a non-arbitrage condition. Note that the deviation from UIP can also be explained with the presence of
} 
as $f_{t}=m_{t}-m_{t}^{*}-\phi\left(y_{t}-y_{t}^{*}\right)$ and define $\lambda=\alpha /(1+\alpha)$. By putting together conditions (5), (6) and (7) and using PPP (eq. 2), a first-order difference equation with a familiar solution for the dynamics the exchange rate can be easily derived:

$$
s_{t}=(1-\lambda)\left[f_{t}+\mathbb{E}_{t} \sum_{j=1}^{\infty} \lambda^{j} f_{t+j}\right]-\lambda\left[\psi_{t}+\mathbb{E}_{t} \sum_{j=1}^{\infty} \lambda^{j} \psi_{t+j}\right]
$$

where the exchange rate depends on the path of the current and expected fundamentals, the UIP condition and deviations $\psi_{t}$. A general solution of this equation under the hypothesis that agents share the public information and perfectly know the model of the economy implies that expectations about the future values of fundamentals and deviations are homogeneous 15

A more sophisticated and realistic information structure generalizes the common expectations framework to include information heterogeneity as proposed by Bacchetta and Van Wincoop (2006, 2013). Heterogeneity implies that agents may have private trading needs due to liquidity or hedge trade that can generate demand for foreign bond influencing the exchange rate, as indicated by the term $\mathbb{E}_{t} \sum_{j=1}^{\infty} \lambda^{j} \psi_{t+j}$. We hypothesize that trading needs expressed by $\psi$ are unrelated to expectations about future fundamental macroeconomic variables and make the simplifying assumption that $\psi_{t}$ is an i.i.d. process with variance $\sigma_{\psi}^{2}$. We thus assume that $f_{t}$ is a linear combination of two random walk processes $f_{1}$ and $f_{2}$, and then the general process for the fundamental is a random walk as well, $f_{t+1}=f_{t}+\epsilon_{t+1}^{f}$ with the variance of $\epsilon_{t+1}^{f}$ being $\sigma_{f}^{2}$. Substituting equations (2), (5), (6) into (7), we obtain a new equation for the dynamics of the exchange rate:

$$
s_{t}=\lambda \overline{\mathbb{E}}_{t} s_{t+1}+(1-\lambda) f_{t}-\lambda \psi_{t},
$$

where the role played by heterogeneous expectations is clearly identified by the term $\overline{\mathbb{E}}_{t} s_{t+1}$. The current value of the rate thus depends on next period expectations of the other predictors. This is true when new information about fundamentals alters expectations on the future value of the exchange rate. Without loss of generality, we assume that each investor at time $t$ captures a private signal about the fundamental at time $t+1$. In a more general perspective, private signals may involve infinite periods ahead for $n$ predictors. In this case we would observe an infinite system where the exchange rate at time $t$ depends

information on fundamentals enclosed in order flow as in Evans (2010) and Chinn and Moore (2011). We abstract from a specific modeling of this factor and consider it an unknown stochastic process in the empirical analysis.

${ }^{15}$ For the functional form of the solution in case of rational expectations, see Bacchetta and Van Wincoop (2012). 
on the fundamental at time $t$ and the average expectation at $t$ of the fundamental at time $t+1$ and so on Bacchetta and Van Wincoop, 2006) 16

In our model, however, heterogeneous one step ahead expectation for each time period is the appropriate model to perform an empirical analysis based on actual data. In the rest of this section, first we describe a social learning model about the choices of professional forecasters, then we integrate it in the standard macro monetary economic model described above.

\subsection{Social learning on exchange rates}

Let us suppose a two-period economy populated by a finite series of predictors, $n=\{1, \ldots, N\}$. In period $t$, each agent $i$ observes noisy private and public signals about the exchange rate $s_{t}$ which belongs to a set $\Psi: s \in \Psi$ and evolves according to the stochastic process:

$$
s_{t}=s_{t-1}+\gamma_{t} \quad \text { where } \quad \gamma_{t} \sim N\left(0, \sigma_{\gamma}^{2}\right) \quad \text { and precision } \quad \rho_{s} \equiv \sigma_{\gamma}^{-2}
$$

where the shock $\gamma_{t}$ occurring at the beginning of period $t$ is normally distributed with mean 0 , variance $\sigma_{\gamma}^{2}$, and precision $\rho_{s} \equiv \sigma_{\gamma}^{-2}$. After the realization of a shock, each agent $i$ receives a common public signal about the fundamental $f_{t}$ as a function of the exchange rate:

$$
f_{t}=s_{t}+\eta_{t} \quad \eta_{t} \sim N\left(0, \sigma_{\eta}^{2}\right) \quad \text { and precision } \quad \rho_{f} \equiv 1 / \sigma_{\eta}^{2}
$$

and a private personal signal:

$$
x_{i t}=s_{t}+\epsilon_{i t} \quad \epsilon_{i t} \sim N\left(0, \sigma_{\epsilon}^{2}\right) \quad \text { and precision } \quad \rho_{x_{i}} \equiv 1 / \sigma_{\epsilon}^{2} .
$$

So while information on the fundamental $f_{t}$ is common knowledge among agents, the private signal $x_{i t}$ is idiosyncratic to agent $i$ and not observed by the other predictors. The common posterior about $s_{t}$, taking into account public information, is therefore normally distributed with mean $\mathbb{E}\left[s_{t} \mid f_{t}\right]=\frac{\rho_{s} s_{t-1}+\rho_{f} f_{t}}{\rho_{s}+\rho_{f}}$ and precision $\rho\left[s_{t} \mid f_{t}\right]=\rho_{s}+\rho_{f}$. For notational simplicity, we denote the mean and the precision of the posterior distribution, respectively as $\tilde{y}_{t}=\mathbb{E}\left[s_{t} \mid f_{t}\right]$ and $\rho_{\tilde{y}}=\rho\left[s_{t} \mid f_{t}\right]$.

Private posteriors are defined by mean $\mathbb{E}\left[s_{t} \mid f_{t}, x_{i t}\right]=\frac{\rho_{\tilde{y}} \tilde{y}_{t}+\rho_{x_{i}} x_{i t}}{\rho_{\tilde{y}}+\rho_{x_{i}}}$ and precision $\rho\left[s_{t} \mid f_{t}, x_{i t}\right]=\rho_{\tilde{y}}+\rho_{x_{i}}$. The weight of public signal in the Bayesian projection of $s$ on the information set $H_{i}(t)=\left\{f_{t} ; x_{i t}\right\}$ is

\footnotetext{
${ }^{16}$ See Townsend $(1983)$.
} 
$\alpha_{\tilde{y}}=\frac{\rho_{\tilde{y}}}{\rho_{\tilde{y}}+\rho_{x_{i}}}$, while the weight of the private one is $\alpha_{x_{i}}=\frac{\rho_{x_{i}}}{\rho_{\tilde{y}}+\rho_{x_{i}}}$. The posterior mean for each agent $i$ is then derived, i.e., $\mathbb{E}_{i}\left(s_{t}\right)=\alpha_{x_{i}} x_{i t}+\alpha_{\tilde{y}} \tilde{y}_{t}$.

Let $e_{i t}=\mathbb{E}_{i t}\left(s_{t+1}\right) \in \mathbb{R}$ denote the predictor $i$ 's expected evaluation on exchange rates, while $\bar{e}_{t} \equiv$ $\int_{j} e_{j t} d j$ and $\sigma_{e}^{2} \equiv \int_{j}\left[e_{i t}-\bar{e}_{t}\right]^{2} d j$ are respectively the mean and the dispersion of investor's expected evaluations in the economy. Each predictor's preferences are explicitly characterized by the following concave increasing function:

$$
U\left(e_{i t}, \bar{e}_{t}, \sigma_{e}^{2}, s_{t}\right)
$$

As general as possible, we assume that the dispersion $\sigma_{e}$ has only a second-order non strategic effect, i.e., $U_{e \sigma}=U_{K \sigma}=U_{s \sigma}=0$, while $U_{\sigma}\left(e_{i t}, \bar{e}_{t}, 0, s_{t}\right)=0, \forall e_{i t} ; \bar{e}_{t} ; s_{t}$. Under perfect information on the exchange rate $s_{t}$, due to symmetry $\left(e_{i t}=\bar{e}_{t}=s_{t}, \forall i\right)$, the best response is given by the unique equilibrium characteristics where predictors' choice exactly coincides with her expectation. In the case of imperfect information instead optimality requires that for any $\left(x_{i t}, f_{t}\right)$, the predictor's choice $e_{i t}=e_{i t}\left(x_{i t} ; f_{t} ; \rho_{\tilde{y}} ; \rho_{x_{i}}\right)$ is such that:

$$
\mathbb{E}\left[U_{e}\left(e_{i}, \bar{e}, \sigma_{e}^{2}, s\right) \mid x_{i t} ; f_{t} ; \rho_{\tilde{y}} ; \rho_{x_{i}}\right]=0, \quad \forall i, t
$$

In the case of a finite number of investors (as in Marinovic et al., 2011), individual's expected utility assumes the following form 17

$$
U\left(e_{i t}, \bar{e}_{t}, \sigma_{e}^{2}, s_{t}\right)=-(1-\delta)\left(e_{i t}-s_{t}\right)^{2}-\delta\left(e_{i t}-\bar{e}_{t}\right)^{2}
$$

The first component is a quadratic loss in the distance between the optimal choice $e_{i}$ and the fundamental $s_{t}$, while the second component is a quadratic loss in the distance between the choice $e_{i t}$ and the average $\bar{e}_{t}$. Each predictor wants to minimize the expected distance between their evaluation and the average. The parameter $\delta \in(0,1)$ is a scalar that parametrizes the intensity of the coordination motive, i.e., the importance that agent $i$ assigns to the expectations of the other predictors of the market.

More intuitively, eq. 15) describes the predictor's process in terms of the decision between two incentives. They constitute the reward rule judging agent's forecast success. The first incentive induces the agent to anchor his/her predictions on the fundamentals. It relies on the distance between the actual spot and the action of the agent and represents the cost of the forecast error, i.e., the cost of making a mistake

\footnotetext{
${ }^{17}$ See appendix AppendixA for the case of a continuum of investors.
} 
with respect to the fundamental. Due to the second incentive, instead, predictors guess the opponents' beliefs because it expresses the cost of distancing from the prediction of the consensus. This is the factor associated with the presence of higher order beliefs and whose weight is expressed by the parameter $\delta$. The quadratic specification of the utility function ensures the linearity of the predictors' best responses and the efficient allocations. Solving for $e_{i t}$, we obtain that:

$$
e_{i t}\left(x_{i} ; f ; \rho_{\tilde{y}} ; \rho_{x_{i}}\right)=(1-\delta) \mathbb{E}_{i}\left[s_{t} \mid x_{i t} ; f_{t} ; \rho_{\tilde{y}} ; \rho_{x_{i}}\right]+\delta \mathbb{E}\left[\bar{e}_{t} \mid x_{i t} ; f_{t} ; \rho_{\tilde{y}} ; \rho_{x_{i}}\right]
$$

which can be rewritten as:

$$
e_{i t}\left(x_{i} ; f ; \rho_{\tilde{y}} ; \rho_{x_{i}}\right)=(1-\delta) \mathbb{E}_{i}\left[s_{t} \mid x_{i t} ; f_{t} ; \rho_{\tilde{y}} ; \rho_{x_{i}}\right]+\delta \frac{e_{i t}}{n}+\delta \mathbb{E}_{i}\left[e_{-i t} \mid x_{i t} ; f_{t} ; \rho_{\tilde{y}} ; \rho_{x_{i}}\right]
$$

where $\mathbb{E}_{i}\left[e_{-i t} \mid x_{i t} ; f_{t} ; \rho_{\tilde{y}} ; \rho_{x_{i}}\right]=\mathbb{E}\left[\left(\frac{e_{1 t}+\ldots+e_{i-1 t}+e_{i+1 t}+\ldots e_{n t}}{n}\right) \mid x_{i t} ; f_{t} ; \rho_{\tilde{y}} ; \rho_{x_{i}}\right]$. In the unique equilibrium with heterogeneous information, each individual $i \neq j$ at time $t$ follows a linear strategy $e_{i t}=e_{t}\left(x_{t} ; f_{t} ; \rho_{\tilde{y}} ; \rho_{x_{i}}\right)$ with:

$$
e_{t}\left(x ; f ; \rho_{\tilde{y}} ; \rho_{x_{i}}\right)=\varphi_{x} x_{t}+\varphi_{\tilde{y}} \tilde{y}_{t}
$$

where $\tilde{y}_{t}=\mathbb{E}\left[s_{t} \mid f_{t}\right]=\frac{\rho_{s} s_{t-1}+\rho_{f} f_{t}}{\rho_{s}+\rho_{f}}$. According to this strategy, the predictor's expectation about the other $(n-1)$ agents is linear in $\left(s_{t} ; f_{t}\right)$ and is given by:

$$
\begin{aligned}
\mathbb{E}_{i}\left[e_{-i t} \mid x_{i t} ; f_{t} ; \rho_{\tilde{y}} ; \rho_{x_{i}}\right] & =\mathbb{E}_{i}\left[\varphi_{x} x_{-i t}+\varphi_{\tilde{y}} \tilde{y}_{t}\right] \\
& =\varphi_{x} \mathbb{E}_{i}\left[s_{t}+\epsilon_{-i t}\right]+\varphi_{\tilde{y}} \tilde{y}_{t} \\
& =\varphi_{x} \mathbb{E}_{i}\left[s_{t}\right]+\varphi_{\tilde{y}} \tilde{y}_{t} .
\end{aligned}
$$

Plugging this expression into predictor's $i$ best response,

$$
e_{i t}=(1-\delta) \mathbb{E}_{i}\left[s_{t} \mid x_{i t} ; f_{t} ; \rho_{\tilde{y}} ; \rho_{x_{i}}\right]+\delta \frac{e_{i t}}{n}+\delta \frac{n-1}{n} \mathbb{E}_{i}\left[e_{-i t} \mid x_{i t} ; f_{t} ; \rho_{\tilde{y}} ; \rho_{x_{i}}\right]
$$

and substituting the posterior mean of $s_{t}$, i.e., $\mathbb{E}_{i}\left(s_{t}\right)=\alpha_{x_{i}} x_{i t}+\alpha_{\tilde{y}} \tilde{y}_{t}$, it follows that:

$$
e_{i t}=\left(1-\varrho+\varrho \varphi_{x}\right)\left[\frac{\rho_{x_{i}}}{\rho_{\tilde{y}}+\rho_{x_{i}}} x_{i t}+\frac{\rho_{\tilde{y}}}{\rho_{\tilde{y}}+\rho_{x_{i}}} \tilde{y}_{t}\right]+\varrho \varphi_{\tilde{y}} \tilde{y}_{t} .
$$


The coefficients $\left(\varphi_{x} ; \varphi_{\tilde{y}}\right)$ for the optimal linear strategy must satisfy:

$$
\varphi_{x}=\frac{(1-\varrho) \rho_{x_{i}}}{(1-\varrho) \rho_{x_{i}}+\rho_{\tilde{y}}} \quad \text { and } \quad \varphi_{\tilde{y}}=\frac{\rho_{\tilde{y}}}{(1-\varrho) \rho_{x_{i}}+\rho_{\tilde{y}}}
$$

as the unique solution of the system, with $\varrho=\frac{n \delta-\delta}{n-\delta}$. The solution of the social learning game relies on the individual expectation about next period exchange rate such that:

$$
\mathbb{E}_{i t}\left(s_{t+1}\right)=\varphi_{x} x_{i t}+\varphi_{y} \tilde{y}_{t}
$$

where the sensitivity of the predictor's expectations to exchange rates is driven by two factors. First, as discussed above, the weight of the beauty contest factor, i.e., $\delta$, identifies the importance assigned to the expectations of other predictors. Note that when $\delta=0$, the best response is given by $e_{i t}=$ $\mathbb{E}\left[s_{t} \mid x_{i t} ; \tilde{m}_{t} ; \rho_{\tilde{y}} ; \rho_{x_{i}}\right]$ so that a predictor's optimal choice coincides with personal expectation. Higher values of $\delta$ induces the agent to take mainly into account public sources of information when making own prediction. Second, the sensitivity of predictor's expectations to the exchange rate depends on the quality of private and public signal in terms of precision. We learn that higher order beliefs places a greater weight to the public signal compared to the private one (Morris and Shin, 2002). Agents put less weight on their own private signal because the public signal acts as a coordinating device in order to predict the actions of others. Using the individual solution of the social learning game as from eq. (23), we aggregate individual predictions among the $n$ investors, such that:

$$
\overline{\mathbb{E}}_{t} s_{t+1}=\varphi_{x} \bar{x}_{t}+\varphi_{y} \tilde{y}_{t}
$$

by substituting it in eq.(9),

$$
s_{t}=\lambda\left(\varphi_{x} \bar{x}_{t}+\varphi_{\tilde{y}} \tilde{y}\right)+(1-\lambda) f_{t}-\lambda \psi_{t}
$$

Knowing that $\tilde{y}_{t}=\mathbb{E}\left[s_{t} \mid f_{t}\right]=\frac{\rho_{s} s_{t-1}+\rho_{f} f_{t}}{\rho_{s}+\rho_{f}}$ and rearranging, we get:

$$
s_{t}=\left(1-\lambda+\lambda \tau_{2} \varphi_{\tilde{y}}\right) f_{t}+\lambda \varphi_{x} \bar{x}_{t}+\lambda \tau_{1} \varphi_{\tilde{y}} s_{t-1}-\lambda \psi_{t}
$$

with $\tau_{1}=\frac{\rho_{s}}{\rho_{s}+\rho_{f}}$ and $\tau_{2}=\frac{\rho_{f}}{\rho_{s}+\rho_{f}}$ that can be reduced to

$$
s_{t}=\beta_{1} f_{t}+\beta_{2} \bar{x}_{t}+\beta_{3} s_{t-1}+\beta_{4} \psi_{t},
$$


where $\beta_{1}=\left(1-\lambda+\lambda \tau_{2} \varphi_{\tilde{y}}\right), \beta_{2}=\lambda \varphi_{x}, \beta_{3}=\lambda \tau_{1} \varphi_{\tilde{y}}$ and $\beta_{4}=-\lambda$. The first term indicates the role of fundamentals in the determination of exchange rate, the second term indicates the role of the strategic interaction of higher order beliefs, the third term represents the role of persistence, the fourth term finally figures out the role of liquidity trade.

\section{Empirical Model}

\subsection{Methods and Data}

According to the exchange rate model à la Bacchetta and Van Wincoop (2006) and introducing a strategic mechanism of interaction as in Ottaviani and Sørensen (2006), we can frame the structural link between the micro and the macro components of our setting as follows:

$$
\begin{aligned}
s_{t} & =\beta_{1} f_{t}+\beta_{2} \bar{x}_{t}+\beta_{3} s_{t-1}+\beta_{4} \psi_{t}+\epsilon_{s, t} \\
f_{t} & =\alpha_{0}+\alpha_{1} f_{1, t}+\alpha_{2} f_{2, t}+\epsilon_{f, t} \\
f_{1, t} & =\phi_{01}+\phi_{11} f_{1, t-1}+\phi_{12} f_{2, t-1}+\epsilon_{f_{1}, t} \\
f_{2, t} & =\phi_{02}+\phi_{21} f_{1, t-1}+\phi_{22} f_{2, t-1}+\epsilon_{f_{2}, t} \\
\tilde{y}_{t} & =\frac{\rho_{f}}{\rho_{f}+\rho_{s}} f_{t}+\left(1-\frac{\rho_{f}}{\rho_{f}+\rho_{s}}\right) s_{t-1} \\
\psi_{t} & =\rho_{\psi} \psi_{t-1}+\epsilon_{\psi, t} \\
\mathbb{E}_{i t}\left[s_{t+1}\right] & =\varphi_{\tilde{y}} \tilde{y}_{t}+\varphi_{x} x_{i, t}, \quad i=1, \ldots, N \\
x_{i, t} & =x_{i, t-1}+\epsilon_{x_{i}, t}, \quad i=1, \ldots, N
\end{aligned}
$$

where both the macro dynamics of exchange rates and the structural parameters of the micro behaviour are described. The shocks $\epsilon_{t}=\left(\epsilon_{s, t}, \epsilon_{f, t}, \epsilon_{f_{1}, t}, \epsilon_{f_{2}, t}, \epsilon_{\psi, t}, \epsilon_{x_{i}, t}\right), i=1, \ldots, N$ are all Gaussian with mean zero and standard deviation, respectively, $\sigma_{s}, \sigma_{f}, \sigma_{f_{1}}, \sigma_{f_{2}}, \sigma_{\psi}$ and, $\sigma_{x_{i}, t}$, whereas $N$ is the number of agents that make predictions on exchange rates.

It is worth noting that eq. (28) corresponds to the structural definition of the exchange rates given in (27), where the coefficients $\beta_{i}$ are functions of the structural parameters. Equations (32) and (34) mimic the learning process defined in Section 3.1. In particular, eq. 32 describes the set of public information in the market due to a combination of past observations on exchange rates and the current value of the fundamental. In turn, eq. (34) identifies the mechanism forming individual expectation as the mixed effect 
of private and public information, weighted respectively by $\varphi_{x}=\frac{(1-\varrho) \rho_{x}}{(1-\varrho) \rho_{x}+\left(\rho_{s}+\rho_{f}\right)}$ and $\varphi_{\tilde{y}}=\frac{\rho_{f}+\rho_{s}}{(1-\varrho) \rho_{x}+\left(\rho_{s}+\rho_{f}\right)}$ with $\varrho=\frac{\delta(N-1)}{N-\delta}$.

We define the dynamics of the fundamentals $f_{t}, f_{1, t}, f_{2, t}$ and the private information flows $x_{i, t}$. The fundamental $f_{t}$ is assumed to be a linear combination of two observable factors $f_{i, t}$ with $i=1,2$, plus an error term. In particular, $f_{i, t}$ are random walks ${ }^{18}$ and influence directly $s_{t}$ and $\tilde{y}_{t}$, while, $\psi_{t}$ is a sequence of $I I D$ shocks, i.e., we set $\rho_{\psi}=0$ to be consistent with the theoretical setup defined in Section 3 . The individual sets of private information, $x_{i, t}$, are unobservable variables with a random-walk structure. This assumption could be in principle relaxed although it is reasonable in this setup due to the non-stationary nature of the exchange rates, their expectations and most of the determinants (Engel and West, 2005). The model defined in 28,35 can be rewritten in compact form as

$$
\Gamma_{0} \mathbf{x}_{t}=c_{x}+\Gamma_{1} \mathbf{x}_{t-1}+\Gamma_{\epsilon} \boldsymbol{\epsilon}_{t}
$$

and in particular $\mathbf{x}_{t}=\left(s_{t}, f_{t}, f_{1, t}, f_{2, t}, \tilde{y}_{t}, \psi_{t}, \mathbb{E}_{i t}, x_{i, t}\right), i=1, \ldots, N$, while $\Gamma_{0}, \Gamma_{1}$ and $\Gamma_{\epsilon}$ are appropriate square matrices of parameters that define the system 28 35. By pre-multiplying eq. 36 with $\Gamma_{0}^{-1}$ we get

$$
\mathbf{x}_{t}=\Theta_{c}+\Theta_{x} \mathbf{x}_{t-1}+\Theta_{\epsilon} \boldsymbol{\epsilon}_{t}
$$

Some of the variables described through eq. 36 are potentially unobservable. For our empirical analysis, we consider as observables the expectations $\mathbb{E}_{i t}\left[s_{t+1}\right]$ which are represented by our dataset on heterogeneous survey forecasts concerning the actual exchange rates and the two fundamentals $f_{i, t}, i=1,2$, that is, $\hat{\mathbf{y}}_{t}=\left(\hat{s}_{t}, \hat{f}_{j, t}, \hat{\mathbb{E}}_{i t}\left[s_{t+1}\right]\right)$, with $j=1,2$ and $i=1, \ldots, N$ We evaluate the expectation for $N=15$ institutions which represent the most influential companies providing predictions for exchange rates in the whole market 20

Data on expectations have been obtained from Foreign Exchange Consensus Forecasts $(F E C F)$. In the survey, Consensus Forecast of London, panelists are asked to forecast spot rates for the use against the euro on the second Monday of every month. They are almost 250; and around 40 on average per each publication are identified individually with their names.

\footnotetext{
${ }^{18}$ In our theoretical framework, this hypothesis is necessary to derive eq. 9 . We set $\phi_{11}=\phi_{22}=1$ and $\phi_{21}=\phi_{12}=0$.

${ }^{19}$ We use the symbol ${ }^{\wedge}$ to distinguish observed from theoretical variables.

${ }^{20}$ For details about the exchange rate dealing and how this affects the market concentration in the foreign exchange predictions, see AppendixD
} 
We refer to individual forecast as the forecast within the panel components whose identity is explicitly indicated in the publication. In particular we focus on one-month-ahead forecasts from January 2006 to June 2012. Since the number and the identity of forecasters are not constant, we manage a database implementing a conservative approach. First we collect the predictions of the institutions that appeared at least once among the individual forecasts in the time span. Then, we assemble all available forecasts, while recording a missing value when the predictor was either absent in the panel for that month publication or when the company prediction for the month was indicated as ' $n a$ '. Consequently we obtain individual predictions for 15 institutions as described in AppendixD.

Macroeconomic variables have been downloaded from Datastream. According to Fratzscher et al. (2012), monthly spot exchange rates on usd to euro have been computed by averaging over the month daily observations from December 2005 to August 2012.

Related to the macroeconomic model in Section 3, fundamentals are $f_{1, t}=m_{t}-m_{t}^{*}$ and $f_{2, t}=y_{t}-y_{t}^{*}$. In particular $f_{1, t}$ is the difference of the logarithms of the money supply measured by the variable $M 2$ for usd and euro at a monthly frequency; whereas $f_{2, t}$ is the difference of the logarithm of the GDP of $U S A$ and euro area. Quarterly data on GDP have been disaggregated to a monthly frequency using the methodology described by Proietti (2006). On the basis of Golinelli and Parigi (2008), we used as leading indicators, long term interest rates (per cent per annum), harmonized unemployment rate, retail trade and industrial production.21

We thus consider the following measurement equations to link our theoretical model to the real-world economy:

$$
\hat{\mathbf{y}}_{t}=S \mathbf{x}_{t}
$$

where $S$ is a selection matrix that links the actual data set to the macroeconomic structure. Equations (37.38) represent a linear and Gaussian state-space system for which the likelihood can be computed in closed form through the Kalman filter. In particular, eq. (37) relies on the latent structure of the model, or transition equation, while $(38)$ is the so called measurement equation. It is worth noting that our database on subjective forecasts is affected by missing values. This is not a relevant problem, since the Kalman filter predicts missing data and allows for the computation of the likelihood function in a natural way (see Koopman et al., 1999 for a treatment on this point).

\footnotetext{
${ }^{21}$ See AppendixD for details on data for macroeconomic variables.
} 
In order to deal with non-stationary observed data on exchange rates, it is appropriate to use first differences. To take into account this transformation, it is easy to redefine the model as follows

$$
\Delta \hat{\mathbf{y}}_{t} \equiv\left[\begin{array}{c}
\Delta \hat{s}_{t} \\
\Delta \hat{f}_{1, t} \\
\Delta \hat{f}_{2, t} \\
\Delta \hat{\mathbb{E}}_{i t}\left[s_{t+1}\right]
\end{array}\right]+\left[\begin{array}{c}
s_{t}-s_{t-1} \\
f_{1, t}-f_{1, t-1} \\
f_{2, t}-f_{2, t-1} \\
\mathbb{E}_{i t}\left[s_{t+1}\right]-\mathbb{E}_{i t-1}\left[s_{t}\right]
\end{array}\right]+\left[\begin{array}{c}
0 \\
0 \\
0 \\
\gamma_{i}
\end{array}\right], i=1, \ldots, N
$$

where $\gamma_{i}$ are Gaussian measurement errors with standard deviation $\sigma_{E_{i}}$ that might affect the observables. Notice that in eq. (39) we also include the lags of the observed variables which are not specified in the vector $\mathbf{x}_{t}$. To fix the problem it is possible to generalize the vector of the variables of the model as follows

$$
\tilde{\boldsymbol{x}}_{t}=\left(s_{t}, f_{t}, f_{1, t}, f_{2, t}, \tilde{y}_{t}, \psi_{t}, \mathbb{E}_{i t}\left[s_{t+1}\right], x_{i, t}, s_{t-1}, f_{1, t-1}, f_{2, t-1}, \mathbb{E}_{i t-1}\left[s_{t}\right]\right), \quad i=1, \ldots, 15
$$

to finally obtain the reduced form which reads

$$
\begin{aligned}
\Delta \hat{\mathbf{y}}_{t} & =\tilde{S} \tilde{\boldsymbol{x}}_{t}+\tilde{\boldsymbol{\gamma}}_{t} \\
\tilde{\boldsymbol{x}}_{t} & =\tilde{\Theta}_{c}+\tilde{\Theta}_{x} \tilde{\boldsymbol{x}}_{t-1}+\tilde{\Theta}_{\epsilon} \tilde{\boldsymbol{\epsilon}}_{t} .
\end{aligned}
$$

\subsection{Prior distributions and inferential methods}

Our main goal is to jointly estimate the structural and the reduced form parameters. Our first interest is to capture the effect of higher order beliefs on the dynamics of the rate. This is identified by the weight $\delta$ in the decision process of the individual predictor. Our second task is to measure the role of private and public informations to determine the actual expectation. We need to explore the coefficients $\varphi_{x}$ and $\varphi_{\tilde{y}}$ obtained from 22 .

In the theoretical model of Section 3, we show that the coefficient $\varphi_{x}$ measures the relevance of private information in the formation process of expectations, while, $\varphi_{\tilde{y}}$ indicates the relevance of public information. There is also an influence of the value of $\delta$ on the dimensions of $\varphi_{x}$ and $\varphi_{\tilde{y}}$. The higher the value of $\delta$, the greater the weight associated to the public signal with respect to the private one.

We recur to Bayesian estimation methods here, and in particular to Markov chain Monte Carlo algorithms $(M C M C)$, which have proved to be successful in the empirical macroeconomic literature Kim and Pagan, 1995, Canova, 2007). This task can be easily handled using a Random Walk Metropolis 
Hastings algorithm ${ }^{22}$ All of the computations in this paper are based on software written using the $\mathrm{Ox}^{\circledR}$ 7.0 language of Doornik (2001) combined with the state space library ssfpack of Koopman et al. (1999).

Our prior choices on the parameters are summarized in Tables 1 to 3 . Overall, we considered prior densities that match the domain of the structural parameters. In particular, we select a prior distribution for the delta parameter equal to 0.5 (and standard deviation 0.1), consequently assigning an equal weight to the two incentives present in the decision function of our predictors (eq. 15).

A priori, we assume that public and private informations play the same role when agents form their own expectations, i.e., without forcing the model to privilege some sources of information. This guess is consistent with the hypothesis that $\varphi_{x}$ and $\varphi_{\tilde{y}}$ are equal. Since these weights depend on the precision coefficients $\rho_{f}, \rho_{s}$ and $\rho_{x}$, we need to find prior distributions for them and at least on average, set $\varphi_{x}=\varphi_{\tilde{y}}=0.5$. To obtain this result, we set the prior distributions for $\rho_{f}$ and $\rho_{s}$ as Gamma with mean 1 and standard deviation 0.1, whereas $\rho_{x}$ is still Gamma, but with larger expected value, namely, 4 and standard deviation 0.423

The discount factor $\lambda$ is a Beta variable with mean 0.5 and standard deviation 0.1. Furthermore, we assume a weakly informative prior for $\alpha_{1}$ and $\alpha_{2}$ that are both Gaussian with mean 0 and rather large variance with respect to the mean, i.e., 1. Finally the standard deviations of the shocks, including standard deviations of the measurement errors, are rather dispersed, and in particular their standard deviations are quite large with respect to the corresponding expected values. They are Inverse Gamma with mean 0.6 and standard deviation 0.2 .

\section{Posterior estimates and Policy Implications}

Posterior estimates have been obtained by running again the $M C M C$ algorithm for 200.000 iterations with a burn-in of 10.000. That is an adequate choice to remove the dependence on the initial conditions. As usual in macro-econometrics (see An and Schorfheide, 2007), initial conditions have been obtained by maximizing the posterior mode for the parameters. Results are reported in Table 1 to 3 .

In particular, table 1 and figure 1 include posterior estimates of the structural relevant parameters, namely posterior averages and credibility intervals, whereas Figure 1 displays prior versus posterior comparisons.

\footnotetext{
${ }^{22}$ See Robert and Casella (1999, ch. 6-7) for a general treatment on MCMC algorithms and Monte Carlo methods in general

${ }^{23}$ An extensive sensitivity analysis suggests that posterior estimates of $\varphi_{x}$ and $\varphi_{\tilde{y}}$ are robust with respect to this choice.
} 
Table 1: Posterior computation (MCMC) - Structural parameters

\begin{tabular}{lcccccc}
\hline & \multicolumn{3}{c}{ Posterior distribution } & \multicolumn{2}{c}{ Prior information } \\
\hline & Parameter value & Mean & $95 \%$ Cred. Int. & Mean & S.E. & Type \\
\hline$p\left(\beta_{1} \mid \hat{\boldsymbol{y}}\right)$ & $\beta_{1}=1-\lambda+\lambda \varphi_{\tilde{y}} \frac{\rho_{f}}{\rho_{f}+\rho_{s}}$ & 0.2302 & {$[0.170,0.301]$} & & & \\
$p\left(\beta_{2} \mid \hat{\boldsymbol{y}}\right)$ & $\beta_{2}=\lambda \varphi_{x}$ & 0.6489 & {$[0.559,0.724]$} & & & \\
$p\left(\beta_{3} \mid \hat{\boldsymbol{y}}\right)$ & $\beta_{3}=\lambda \varphi_{\tilde{y}} \frac{\rho_{s}}{\rho_{f}+\rho_{s}}$ & 0.1209 & {$[0.070,0.177]$} & & & \\
$p\left(\beta_{4} \mid \hat{\boldsymbol{y}}\right)$ & $\beta_{4}=-\lambda$ & -0.8561 & {$[-0.916,-0.779]$} & & & \\
$p\left(\alpha_{1} \mid \hat{\boldsymbol{y}}\right)$ & & 0.0842 & {$[-0.716,0.851]$} & 0 & 1 & Normal \\
$p\left(\alpha_{2} \mid \hat{\boldsymbol{y}}\right)$ & & -2.5237 & {$[-3.724,-1.289]$} & 0 & 1 & Normal \\
$p\left(\rho_{f} \mid \hat{\boldsymbol{y}}\right)$ & & 0.8764 & {$[0.706,1.070]$} & 1 & 0.1 & Gamma \\
$p\left(\rho_{s} \mid \hat{\boldsymbol{y}}\right)$ & & 1.2342 & {$[1.029,1.454]$} & 1 & 0.1 & Gamma \\
$p\left(\rho_{x} \mid \hat{\boldsymbol{y}}\right)$ & & 3.618 & {$[2.910,4.394]$} & 4 & 0.4 & Gamma \\
$p\left(\varphi_{x} \mid \hat{\boldsymbol{y}}\right)$ & $\varphi_{x}=\frac{(1-\varrho) \rho_{x}}{(1-\varrho) \rho_{x}+\left(\rho_{s}+\rho_{f}\right)}$ & 0.2412 & {$[0.148,0.352]$} & & & \\
$p\left(\varphi_{\tilde{y}} \mid \hat{\boldsymbol{y}}\right)$ & $\rho_{\tilde{y}}=\frac{\rho_{f}+\rho_{s}}{(1-\varrho) \rho_{x}+\left(\rho_{s}+\rho_{f}\right)}$ & 0.7588 & {$[0.648,0.852]$} & & & \\
$p\left(\sigma_{s} \mid \hat{\boldsymbol{y}}\right)$ & & 1.019 & {$[0.468,1.455]$} & 0.6 & 0.16 & Inv. Gamma \\
$p\left(\sigma_{f} \mid \hat{\boldsymbol{y}}\right)$ & & 9.2763 & {$[6.986,12.625]$} & 0.6 & 0.16 & Inv. Gamma \\
$p\left(\sigma_{f} \mid \hat{\boldsymbol{y}}\right)$ & & 0.5854 & {$[0.503,0.683]$} & 0.6 & 0.16 & Inv. Gamma \\
$p\left(\sigma_{f 2} \mid \hat{\boldsymbol{y}}\right)$ & & 0.9472 & {$[0.812,1.116]$} & 0.6 & 0.16 & Inv. Gamma \\
$p\left(\sigma_{\phi} \mid \hat{\boldsymbol{y}}\right)$ & & 0.8621 & {$[0.408,1.588]$} & 0.6 & 0.16 & Inv. Gamma \\
$p(\lambda \mid \hat{\boldsymbol{y}})$ & & 0.8561 & {$[0.779,0.916]$} & 0.5 & 0.1 & Beta \\
$p(\delta \mid \hat{\boldsymbol{y}})$ & & 0.8202 & {$[0.694,0.902]$} & 0.5 & 0.1 & Beta \\
\hline
\end{tabular}

The first interesting result relates to the value of the coefficient $\delta$. A sensitive shift to the right of the posterior is observed in the comparison with its prior distribution confirming the important role of the beauty contest in the predictor's evaluation process. Individuals assign more weight than expected (82\%) of interpreting correctly the other predictors' beliefs and a smaller weight (18\%) to the cost of making forecast error with respect to the fundamental. The rational incentives of predictors are therefore distorted. The value worth of the consensus is definitely higher than the option of making the right choice on the basis of their own private information.

The result is relevant, not only for the correct comprehension of the micro-behavior of predictors, but also because it helps to explain the persistent presence of some inefficiencies in predictions markets. This is confirmed by a long strand of literature on the inefficiency of survey expectations for exchange rates (Cavaglia et al., 1993, Ito, 1990; Frankel and Froot, 1990; MacDonald and Ian, 1996; Mitchell and Pearce, 2007; Pancotto et al. 2014).

Higher cost in the wrong choice on the consensus's evaluation leads to an optimization process with apparently odd conclusions. Predictors may in principle persist in incorrect predictions compared to 

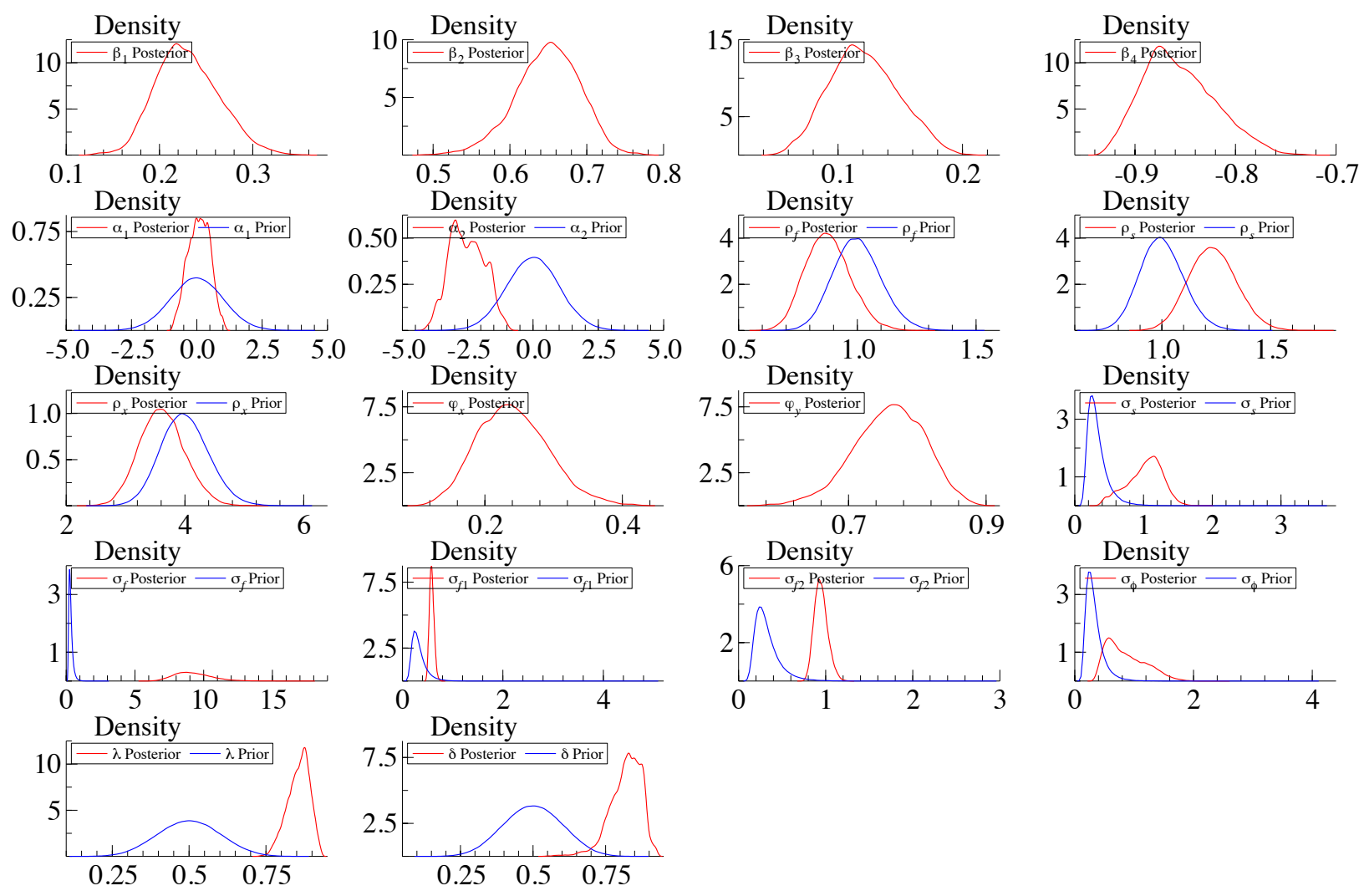

Figure 1: Prior vs. Posterior distributions of the structural parameters

the actual market realization since their choice is in line with what they expect the consensus will do. When this incentive prevails, a potential bias at the aggregate level could be observed in forecasting the exchange rates. A second relevant point is the role that public and private information have on individual forecast. Our analysis is based on the coefficients $\varphi_{x}$ and $\varphi_{\tilde{y}}$. Results suggest that public information accounts for about 75 percent of the predictions, whereas just 25 percent depends on private information. This is coherent with the previous result related to the weight associated with higher order beliefs. The combination between higher order beliefs and information structure ensures rather a rational behavior in the decision problem. When agents care more about the consensus prediction rather than their own personal assessment, they reduce implicitly the importance of their private signal. Robustness checks suggest that this phenomenon is always verified despite ex-ante values of parameters $\varphi_{x}$ and $\varphi_{\tilde{y}}$ are assumed to be equal and independent by the precision of both signals. Although the precision of 
the private signal $\rho_{x}$ is higher than the precisions of the public one, i.e., $\rho_{f}$ and $\rho_{s}$, the role of higher order beliefs is largely confirmed. Public information in a beauty contest environment, therefore, acts as a coordinating device. This is a central result, firstly, proposed by Morris and Shin (2002). We have intentionally integrated it in our framework to test its presence and intensity in the context of the exchange rate market. Furthermore, it also furnishes a complementary result to the empirical test of the scapegoat model of Bacchetta and Van Wincoop (2004) implemented by Fratzscher et al. (2012). They discover that using survey predictions on fundamentals as proxies for scapegoat effects increases the ability to explain exchange rate movements. Public information is, therefore, capable of capturing changes in the actual dynamics of the rate. Our result is closely related to these points. First, we estimate the intensity of beauty contests factor and, as theoretically predicted, we discover that the importance of the role of higher order beliefs is associated to a greater weight assigned on public information. Second, using the result of Fratzscher et al. (2012), we infer that public information that agents overrate can be a distorted information. The predictors are rationally searching for fundamental information, but then they end up in overweighting a public information that is not informative. This is clearly due on one side to the presence of higher order beliefs and on the other side to the uncertainty that the heterogeneity of expectations conveys. The fundamental is therefore transformed into a scapegoat in case of uncertainty about the structural parameters. In particular, the high value of the variance of the fundamental, $p\left(\sigma_{f} \mid \hat{\boldsymbol{y}}\right)$ (around 9) in table 1 suggests a coherence between the short term uncertainty suggested by Fratzscher et al. (2012) and the one derived by the fundamental movements. This exactly generates the scapegoat effect discussed by Bacchetta and Van Wincoop (2004).

\subsection{Robustness checks and goodness-of-fit}

In this section we evaluate the performance of our model against the data. First we compare our model with a rational expectation dynamics. As a benchmark, we consider the rational expectation model that closely mimics the dynamics defined in eq. (9). In particular we consider

$$
s_{t}=\lambda \mathbb{E}_{t}\left[s_{t+1}\right]+(1-\lambda) f_{t}-\lambda \psi_{t}+\epsilon_{t},
$$

in which $f_{t}$ is described in eq. 29, while $\psi_{t}$ is an independent and identically distributed sequence. Furthermore, rational expectations are defined such that $\mathbb{E}_{t}\left[s_{t+1}\right]=s_{t}+\eta_{t}$, where $\eta_{t}$ is a gaussian shock with mean zero and constant variance. Rational expectation dynamics on exchange rates has been estimated 
through MCMC. In particular for each posterior draw of the parameters, we solved the rational expectation system using $\operatorname{Sims}(2002)$ and implementing it with the Ox package LiRE of Mavroeidis and Zwols (2007). Then for each parameter, we simulated the one-step-ahead prediction of the rational expectation dynamics. Figure 2 compares the average rational expectations trajectory and the average one from the

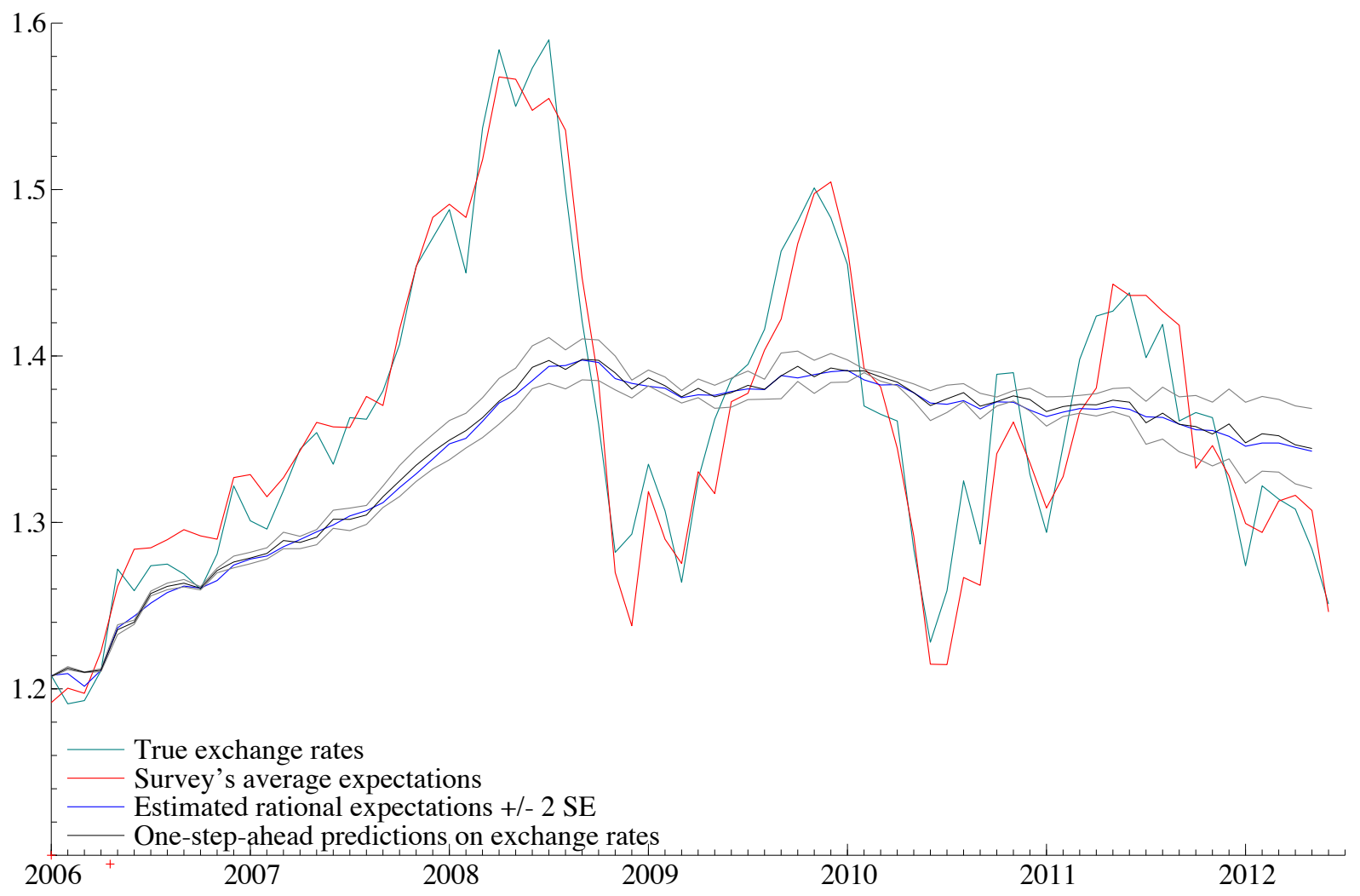

Figure 2: Actual exchange rates (green line), average from the survey (red line) together with an estimate of the rational expectation on exchange rates $\mathrm{E}_{t}\left[s_{t+1}\right]$ (blue line) and the one-step-ahead prediction on $s_{t}$ (black line).

survey ${ }^{24}$ Figure 2 still points out the predicted average expectations. They differ substantially from the observed ones and it emerges that predicted exchange rates do not replicate at all the dynamics of actual exchange rates.

This empirical evidence can be considered as a symptom that rational expectations fail in providing

\footnotetext{
${ }^{24}$ Here rational expectations has been computed as the average trajectory compared to the posterior draws of the model's parameters.
} 
a good fit to actual data. This is the reason why using survey data appears to be a viable strategy to provide a better description of actual exchange rates. As a second exercise, we thus compute the predicted exchange rates and their returns, see figure 3 displaying one-step-ahead prediction for them.

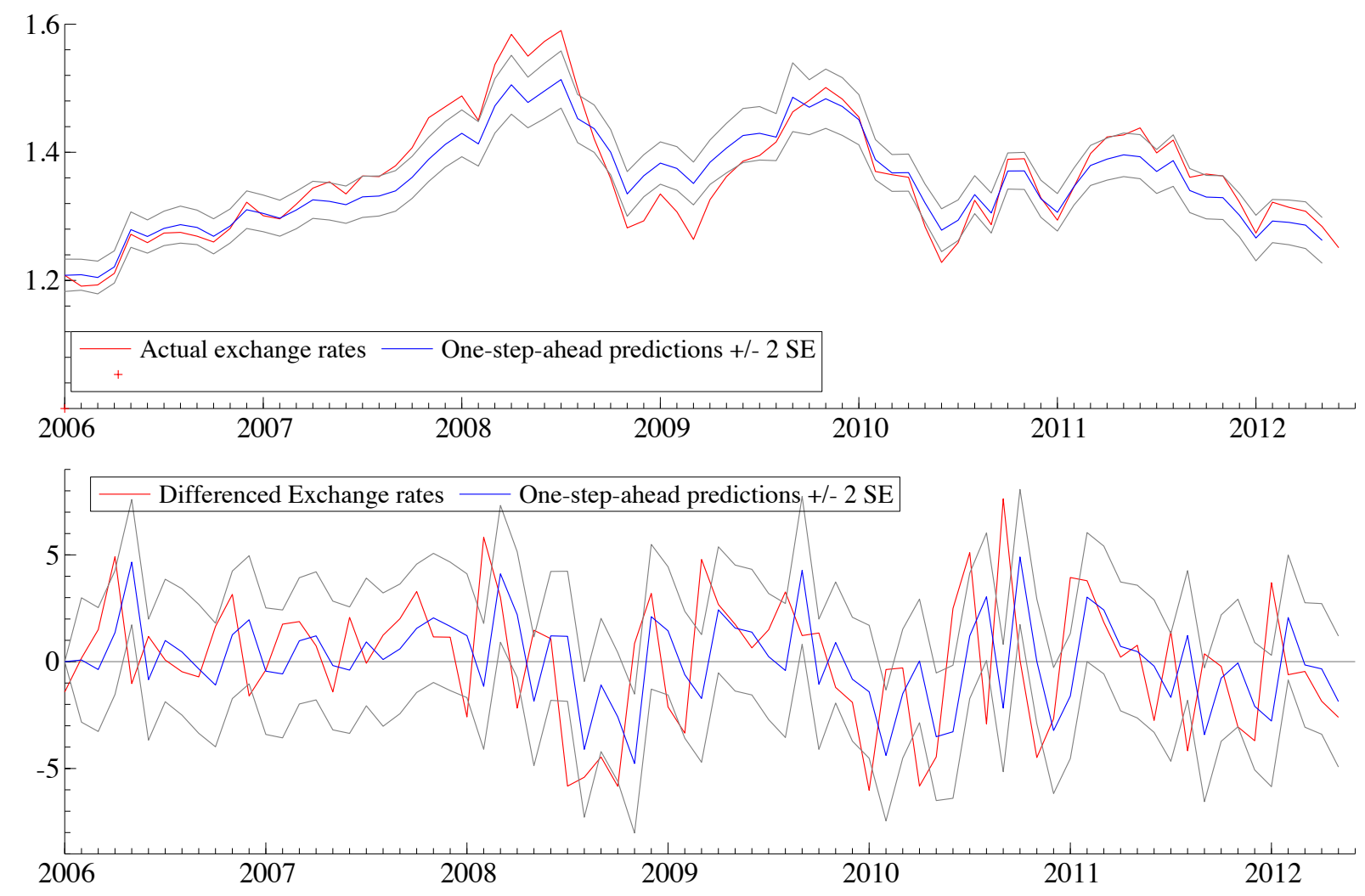

Figure 3: Upper panel: Actual exchange rates (red line) vs. predicted exchange rates (blue line) together with $95 \%$ credibility bands. Lower panel: Actual exchange rates returns (red line) vs. predicted exchange rates returns (blue line) together with $95 \%$ credibility bands.

In this case, actual data are replicated quite accurately by the model. Indeed, more than $70 \%$ of actual exchange rates fall in the $95 \%$ credibility interval. In particular predicted estimates provide a good proxy for the trending behavior of the true data.

We also investigate the role of measurement errors in the model. We thus compare the model with measurement errors together with the estimated model. We evaluate the goodness-of-fit through the marginal likelihood estimated as the harmonic means of the likelihood function evaluated for each posterior draw of the parameter vector (see An and Schorfheide, 2007 for this point). The log-marginal likelihoods 
for the model with and without measurement errors are respectively -2349.2 and the -2359.7 , which imply a Bayes factor of about $e^{10}$ in favor of the former model. This evidence suggests a strong rejection of the model with no measurement errors.

To study the dynamic interactions between the variables in level of the system, it is useful to analyze how exchange rates react to structural shocks. This assessment is illustrated in figure 4 We show the impulse response functions (IRFs, hereafter) of exchange rates due to positive economic shocks, related to exchange rates, fundamentals, liquidity and private information. For each shock, the impulse-response functions are shown along with the 95 percent credible intervals. Despite the non-stationary nature in the dynamics of the model, impulse response functions can still be computed (Lütkepohl, 2005, ch. 6.7).
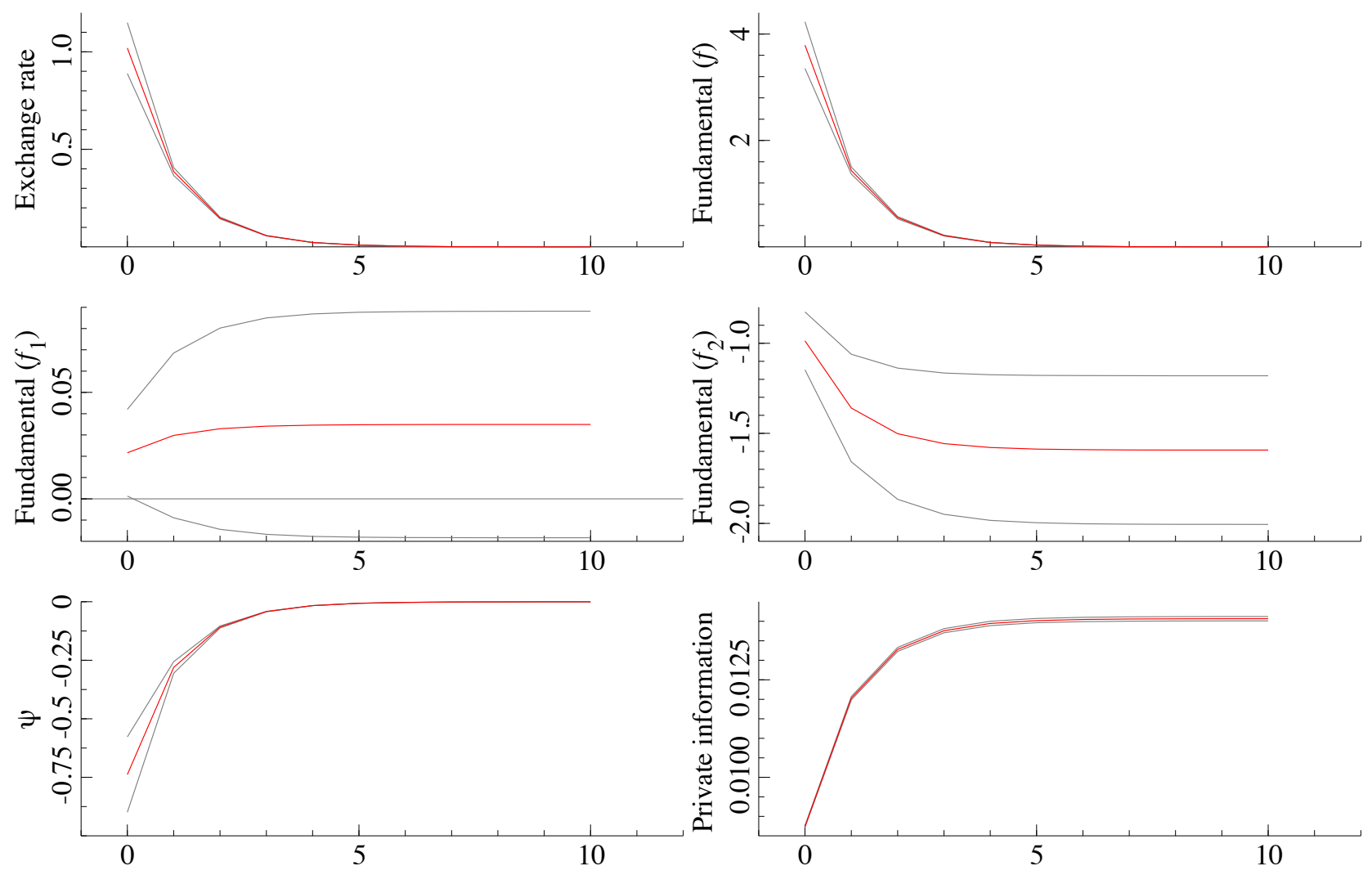

Figure 4: Impulse Response Functions and the 95\% credible intervals

In the graphs of figure 4, red line relies on the IRF, while the grey band is the $95 \%$ credible interval for the IRF. In the top left panel we observe how a shock to exchange rates affects the overall dynamics of the rate for a short period, dying out at the end of the period. The same happens for the shock to the linear 
combination of the two fundamentals (top right panel), which as well affects the dynamics of the rate and then fades away. This is coherent with the standard monetary model with flexible prices where an anticipated monetary shock affect the exchange rate only temporarily. In the bottom left panel we observe that a shock to liquidity generates a substantial effect on the exchange rate dynamics - coherently with Evans and Lyons (2002) - but this effect is absorbed with time because of the choice to model liquidity as a white noise. The increase in the role of private information is observed in the last graph of figure4, particularly in the short run, although the results are not statistically significant.

Finally, we also computed the forecast error variance decomposition (FEVD) for the returns on exchange rates. To compute the FEVD we exploited the cointegration relationships of the system to derive its MA representation of $\Delta \boldsymbol{x}_{t}{ }^{25}$ This representation has been obtained through the Smith-McMillan factorization of the polynomial matrix associated to the model as proposed in Engle and Yoo (1991).

Our analysis suggests that the shocks on fundamentals is the primary factor on explaining $\Delta s_{t}$. In particular fundamentals explain about $90 \%$ of the variance in returns. Shocks on exchange rates explain about $6 \%$ of the variability whereas liquidity appear to be less relevant and counts for about $3 \%$.

Apparently, private informations are not relevant for forecasting purposes. On the other hand, figure 2 suggests that rational expectations by themselves provide poor one-step-ahead predictions. These findings suggests that fundamentals represent the most important device to forecast exchange rates. However excluding the learning process from the model may cause troubles due to some form of mis-specification of the exchange rates dynamics that leads to an unprecise estimate of the model's parameters. Even this results thus provide evidence in favor of our specification versus the standard rational expectation model. Furthermore, to predict returns on exchange rates, measurement errors play no roles at all.

\section{Conclusions}

The paper tends to investigate the process leading to the dynamics of exchange rates. Expectations are formed using a social learning game where agents exploit jointly public and private information using a learning scheme according to Morris and Shin (2002). Our first goal was to verify whether a mechanism of higher order beliefs takes place and its connection with the scapegoat theory of exchange rates (Bacchetta and Van Wincoop, 2004). Our second task was to quantify through a structural time series model the connections between public and private informations and their role in building expectations. Results show

\footnotetext{
${ }^{25}$ See AppendixC for some technical details.
} 
that higher order beliefs are relevant in forming agent's expectations. Furthermore, public information plays a crucial role as a coordination device to generate expectations among agents on the basis of their forecasting abilities. As a side result, we also noticed that rational expectations appear to provide poor one-step-ahead predictions for exchange rates.

Table 2: Posterior computation (MCMC) - Other parameters

\begin{tabular}{lccccc}
\hline & \multicolumn{3}{c}{ Posterior distribution } & \multicolumn{3}{c}{ Prior information } \\
\hline & Mean & $95 \%$ Cred. Int. & Mean & S.E. & Type \\
$p\left(\sigma_{x_{1}} \mid \hat{\boldsymbol{y}}\right)$ & 0.6314 & {$[0.395,1.081]$} & 0.6 & 0.16 & Inv. Gamma \\
$p\left(\sigma_{x_{2}} \mid \hat{\boldsymbol{y}}\right)$ & 0.6096 & {$[0.393,0.972]$} & 0.6 & 0.16 & Inv. Gamma \\
$p\left(\sigma_{x_{3}} \mid \hat{\boldsymbol{y}}\right)$ & 0.6044 & {$[0.375,0.975]$} & 0.6 & 0.16 & Inv. Gamma \\
$p\left(\sigma_{x_{4}} \mid \hat{\boldsymbol{y}}\right)$ & 0.6098 & {$[0.392,0.977]$} & 0.6 & 0.16 & Inv. Gamma \\
$p\left(\sigma_{x_{5}} \mid \hat{\boldsymbol{y}}\right)$ & 0.6467 & {$[0.393,1.104]$} & 0.6 & 0.16 & Inv. Gamma \\
$p\left(\sigma_{x_{6}} \mid \hat{\boldsymbol{y}}\right)$ & 0.5871 & {$[0.376,0.958]$} & 0.6 & 0.16 & Inv. Gamma \\
$p\left(\sigma_{x_{7}} \mid \hat{\boldsymbol{y}}\right)$ & 0.5902 & {$[0.372,1.125]$} & 0.6 & 0.16 & Inv. Gamma \\
$p\left(\sigma_{x_{8}} \mid \hat{\boldsymbol{y}}\right)$ & 0.6077 & {$[0.371,1.104]$} & 0.6 & 0.16 & Inv. Gamma \\
$p\left(\sigma_{x_{9}} \mid \hat{\boldsymbol{y}}\right)$ & 0.5806 & {$[0.372,0.934]$} & 0.6 & 0.16 & Inv. Gamma \\
$p\left(\sigma_{x_{10}} \mid \hat{\boldsymbol{y}}\right)$ & 0.6039 & {$[0.386,0.963]$} & 0.6 & 0.16 & Inv. Gamma \\
$p\left(\sigma_{x_{11}} \mid \hat{\boldsymbol{y}}\right)$ & 0.6247 & {$[0.381,1.017]$} & 0.6 & 0.16 & Inv. Gamma \\
$p\left(\sigma_{x_{12}} \mid \hat{\boldsymbol{y}}\right)$ & 0.6142 & {$[0.380,1.056]$} & 0.6 & 0.16 & Inv. Gamma \\
$p\left(\sigma_{x_{13}} \mid \hat{\boldsymbol{y}}\right)$ & 0.5919 & {$[0.391,0.913]$} & 0.6 & 0.16 & Inv. Gamma \\
$p\left(\sigma_{x_{14}} \mid \hat{\boldsymbol{y}}\right)$ & 0.6243 & {$[0.404,0.983]$} & 0.6 & 0.16 & Inv. Gamma \\
$p\left(\sigma_{x_{15}} \mid \hat{\boldsymbol{y}}\right)$ & 0.6564 & {$[0.406,1.183]$} & 0.6 & 0.16 & Inv. Gamma \\
\hline
\end{tabular}

Table 3: Posterior computation (MCMC) - Measurement Errors

\begin{tabular}{lccccl}
\hline & \multicolumn{3}{c}{ Posterior distribution } & \multicolumn{2}{c}{ Prior information } \\
\hline & Mean & $95 \%$ Cred. Int. & Mean & S.E. & Type \\
$p\left(\sigma_{E_{1}} \mid \hat{\boldsymbol{y}}\right)$ & 1.4055 & {$[1.170,1.686]$} & 0.6 & 0.16 & Inv. Gamma \\
$p\left(\sigma_{E_{2}} \mid \hat{\boldsymbol{y}}\right)$ & 1.7511 & {$[1.386,2.204]$} & 0.6 & 0.16 & Inv. Gamma \\
$p\left(\sigma_{E_{3}} \mid \hat{\boldsymbol{y}}\right)$ & 2.5709 & {$[2.162,3.071]$} & 0.6 & 0.16 & Inv. Gamma \\
$p\left(\sigma_{E_{4}} \mid \hat{\boldsymbol{y}}\right)$ & 3.9357 & {$[3.343,4.640]$} & 0.6 & 0.16 & Inv. Gamma \\
$p\left(\sigma_{E_{5}} \mid \hat{\boldsymbol{y}}\right)$ & 0.867 & {$[0.604,1.218]$} & 0.6 & 0.16 & Inv. Gamma \\
$p\left(\sigma_{E_{6}} \mid \hat{\boldsymbol{y}}\right)$ & 3.3454 & {$[2.844,3.961]$} & 0.6 & 0.16 & Inv. Gamma \\
$p\left(\sigma_{E_{7}} \mid \hat{\boldsymbol{y}}\right)$ & 2.8154 & {$[2.346,3.380]$} & 0.6 & 0.16 & Inv. Gamma \\
$p\left(\sigma_{E_{8}} \mid \hat{\boldsymbol{y}}\right)$ & 1.5442 & {$[1.293,1.841]$} & 0.6 & 0.16 & Inv. Gamma \\
$p\left(\sigma_{E_{9}} \mid \hat{\boldsymbol{y}}\right)$ & 2.6808 & {$[2.277,3.157]$} & 0.6 & 0.16 & Inv. Gamma \\
$p\left(\sigma_{E_{10}} \mid \hat{\boldsymbol{y}}\right)$ & 1.9843 & {$[1.693,2.337]$} & 0.6 & 0.16 & Inv. Gamma \\
$p\left(\sigma_{E_{11}} \mid \hat{\boldsymbol{y}}\right)$ & 2.9299 & {$[2.456,3.525]$} & 0.6 & 0.16 & Inv. Gamma \\
$p\left(\sigma_{E_{12}} \mid \hat{\boldsymbol{y}}\right)$ & 2.9091 & {$[2.450,3.444]$} & 0.6 & 0.16 & Inv. Gamma \\
$p\left(\sigma_{E_{13}} \mid \hat{\boldsymbol{y}}\right)$ & 2.5205 & {$[2.148,2.969]$} & 0.6 & 0.16 & Inv. Gamma \\
$p\left(\sigma_{E_{1}} \mid \hat{\boldsymbol{y}}\right)$ & 2.9644 & {$[2.542,3.491]$} & 0.6 & 0.16 & Inv. Gamma \\
$p\left(\sigma_{E_{15}} \mid \hat{\boldsymbol{y}}\right)$ & 1.687 & {$[1.410,2.014]$} & 0.6 & 0.16 & Inv. Gamma \\
\hline
\end{tabular}




\section{References}

Allen, F., Morris, S., Shin, H., 2006. Beauty contests and iterated expectations in asset markets. Review of Financial Studies 19 (3), 719-752.

An, S., Schorfheide, F., 2007. Bayesian analysis of dsge models. Econometric Reviews 26, 113-172.

Angeletos, G., La'O, J., 2009. Noisy business cycles. NBER Macroeconomics Annual 24 (1), 319-378.

Angeletos, G., Lorenzoni, G., Pavan, A., 2012. Wall street and silicon valley: A delicate interaction. North-Western working paper.

Avraham, D., Ungar, M., Zilberfarb, B.-Z., 1987. Are foreign exchange forecasts rational?: An empirical note. Economics Letters 24 (3), $291-293$.

Bacchetta, P., Van Wincoop, E., 2004. A scapegoat model of exchange-rate fluctuations. American Economic Review 94 (2), 114-118.

Bacchetta, P., Van Wincoop, E., 2006. Can information heterogeneity explain the exchange rate determination puzzle? The American Economic Review 96 (3), 552-576.

Bacchetta, P., Van Wincoop, E., 2012. Modeling exchange rates with incomplete information. In: J. James, I. M., L.Sarno (Eds.), Handbook of Exchange Rates. Wiley Handbook Series, pp. 375-389.

Bacchetta, P., Van Wincoop, E., 2013. On the unstable relationship between exchange rates and macroeconomic fundamentals. Journal of International Economics 91 (1), 18-26.

Benassy-Quere, A., Larribeau, S., MacDonald, R., 2003. Models of exchange rate expectations: how much heterogeneity? Journal of International Financial Markets, Institutions and Money 13 (2), 113-136.

Canova, F., 2007. Methods for Applied Macroeconomic Research. Princeton University Press, New Jersey.

Cavaglia, S., Verschoor, W. F. C., Wolff, C. P., 1993. Further evidence on exchange rate expectations. Journal of International Money and Finance 12 (1), 78-98.

Cerra, V., Saxena, S. C., 2010. The monetary model strikes back: Evidence from the world. Journal of International Economics 81 (2), 184-196. 
Cheung, Y.-W., Chinn, M. D., Pascual, A. G., 2005. Empirical exchange rate models of the nineties: Are any fit to survive? Journal of International Money and Finance 24 (7), 1150-1175.

Chinn, M., Frankel, J., 1994. Patterns in exchange rate forecasts for 25 currencies. Tech. rep., National Bureau of Economic Research.

Chinn, M. D., Meese, R. A., 1995. Banking on currency forecasts: How predictable is change in money? Journal of International Economics 38 (1), 161-178.

Chinn, M. D., Moore, M. J., 2011. Order flow and the monetary model of exchange rates: Evidence from a novel data set. Journal of Money, Credit and Banking 43 (8), 1599-1624.

Chionis, D., MacDonald, R., 1997. Some tests of market microstructure hypotheses in the foreign exchange market. Journal of Multinational Financial Management 7 (3), 203-229.

Devereux, M., Engel, C., 2002. Exchange rate pass-through, exchange rate volatility, and exchange rate disconnect. Journal of Monetary Economics 49 (5), 913-940.

Dominguez, K., 1986. Are foreign exchange forecasts rational?: New evidence from survey data. Economics Letters $21(3), 277-281$.

Doornik, J., 2001. Ox: An Object-Oriented Matrix Programming Language. Timberlake Consultants Press, London.

Elliott, G., Ito, T., April 1999. Heterogeneous expectations and tests of efficiency in the yen/dollar forward exchange rate market. Journal of Monetary Economics 43 (2), 435-456.

Engel, R., West, K. D., 2005. Exchange rates and fundamentals. Journal of Political Economy 113 (3), $485-517$.

Engle, R., Yoo, B., 1991. Cointegrated economic time series: An overview with new results. In: Engle, R., Granger, C. (Eds.), Long-Run Economic Relationship: Readings in Cointegration. Oxford University Press, pp. 237-266.

Evans, M., Lyons, R., 2002. Order flow and exchange rates dynamics. Journal of Political Economy 110 (1), 170-180. 
Evans, M., Lyons, R., 2005. Meese-rogoff redux: Micro-based exchange rate forecasting. American Economic Review 95 (2), 405-414.

Evans, M. D., 2010. Order flows and the exchange rate disconnect puzzle. Journal of International Economics 80 (1), 58-71.

Faust, J., Rogers, J. H., H Wright, J., 2003. Exchange rate forecasting: the errors weve really made. Journal of International Economics 60 (1), 35-59.

Frankel, J. A., Froot, K. A., 1990. Chartists, fundamentalists, and trading in the foreign exchange market. The American Economic Review, 181-185.

Fratzscher, M., Sarno, L., Zinna, G., 2012. The scapegoat theory of exchange rates: the first tests. Working paper series, no. 1418, feb. 2012, European Central Bank.

Froot, K., Ramadorai, T., 2005. Currency returns, intrinsic value, and institutional-investor flows. Journal of Finance $60(3), 1535-1566$.

Golinelli, R., Parigi, G., 2008. Real-time squared: A real-time data set for real-time gdp forecasting. International Journal of Forecasting 24 (3), 368-385.

Groen, J. J., 2005. Exchange rate predictability and monetary fundamentals in a small multi-country panel. Journal of Money, Credit and Banking, 495-516.

Harsanyi, J., 1967. Games with incomplete information played by bayesian players, i-iii part i. the basic model. Management Science 14 (3), 159-182.

Husted, S., MacDonald, R., 1998. Monetary-based models of the exchange rate: a panel perspective. Journal of International Financial Markets, Institutions and Money 8 (1), 1-19.

Ito, T., 1990. Foreign exchange rate expectations: Micro survey data. The American Economic Review $80(3), 434-49$.

James, J., Marsh, I., Sarno, L., 2012. Handbook of Exchange Rates. Vol. 2. John Wiley \& Sons.

Jongen, R., Verschoor, W. F., Wolff, C. C., 2008. Foreign exchange rate expectations: survey and synthesis. Journal of Economic Surveys 22 (1), 140-165. 
Keynes, J. M., 1936. The General Theory of Employment, Interest and Money. Independent Publishing Platform.

Kim, K., Pagan, A., 1995. Econometric analysis of calibrated macroeconomic models. In: Pesaran, H., Wickens., M. (Eds.), Handbook of Applied Econometrics: Macroeconomics. Basil Blackwell, Oxford, pp. $356-390$.

Kim, S., Shephard, N., Chib, S., 1998. Stochastic volatility: Likelihood inference and comparison with arch models. Review of Economic Studies 65, 361-393.

Koopman, S., Shephard, N., Doornik, J. A., 1999. Statistical algorithms for models in state space using ssfpack 2.2. Econometrics Journal 2, 113-166.

Lütkepohl, H., 2005. New Introduction to Multiple Time Series Analysis. Springer-Verlag Berlin Heidelberg.

MacDonald, R., Ian, W., 1996. Currency forecasters are heterogeneous: confirmation and consequences. Journal of International Money and Finance 15 (5), 665-685.

Marinovic, I., Ottaviani, M., Srensen, P., 2011. Modeling idea markets: Between beauty contests and prediction markets. In: Williams, L. V. (Ed.), Prediction Markets: Theory and Applications. Routledge, pp. $4-17$.

Mark, N., 1995. Exchange rates and fundamentals; evidence on long-horizon predictability. American Economic Review 85 (1), 201-218.

Mark, N. C., Sul, D., 2001. Nominal exchange rates and monetary fundamentals: evidence from a small post-bretton woods panel. Journal of International Economics 53 (1), 29-52.

Mavroeidis, S., Zwols, Y., 2007. Lire an ox package for solving linear rational expectations models version 3.0. Tech. rep., Tech. rep., Brown University, working paper.

Meese, R. A., Rogoff, K., 1983. Empirical exchange rate models of the seventies: Do they fit out of sample? Journal of International Economics 14 (12), 3 - 24.

Mitchell, K., Pearce, D. K., 2007. Professional forecasts of interest rates and exchange rates: Evidence from the wall street journals panel of economists. Journal of Macroeconomics 29 (4), 840-854. 
Molodtsova, T., Nikolsko-Rzhevskyy, A., Papell, D. H., 2008. Taylor rules with real-time data: A tale of two countries and one exchange rate. Journal of Monetary Economics 55, S63-S79.

Molodtsova, T., Nikolsko-Rzhevskyy, A., Papell, D. H., 2011. Taylor rules and the euro. Journal of Money, Credit and Banking 43 (2-3), 535-552.

Molodtsova, T., Papell, D. H., 2009. Out-of-sample exchange rate predictability with taylor rule fundamentals. Journal of International Economics 77 (2), 167-180.

Morris, S., Shin, H. S., 2002. The social value of public information. American Economic Review 92 (5), $1521-1534$.

Myatt, D., Wallace, C., 2012. Endogenous information acquisition in coordination games. Review of Economic Studies 79 (1), 340-374.

Myatt, D., Wallace, C., 2014a. Central bank communication design in a lucas-phelps economy. Journal of Monetary Economics 63 (1), 64-79.

Myatt, D., Wallace, C., 2014b. Cournot competition and the social value of information. Journal of Economic Theory, forthcoming.

O’Hara, M., 1995. Market Microstructure Theory. Blackwell, Oxford.

Ottaviani, M., Sørensen, P. M., 2006. The strategy of professional forecasting. Journal of Financial Economics $81(2), 441-466$.

Pancotto, F., Pericoli, F. M., Pistagnesi, M., 2014. Overreaction in survey exchange rate forecasts. Journal of Forecasting 33, 243-258.

Payne, R., 2003. Informed trade in spot foreign exchange markets: An empirical investigation. Journal of International Economics 61 (2), 307-330.

Pedroni, P., 1999. Critical values for cointegration tests in heterogeneous panels with multiple regressors. Oxford Bulletin of Economics and statistics 61 (S1), 653-670.

Proietti, T., 2006. Temporal disaggregation by state space methods: Dynamic regression methods revisited. Econometrics Journal 9, 357-372. 
Robert, C., Casella, G., 1999. Monte Carlo Statistical Methods. Springer, Berlin.

Sims, C., 2002. Solving linear rational expectations models. Computational Economics 20 (1-2), 1-20.

Townsend, R. M., 1983. Forecasting the forecasts of others. The Journal of Political Economy 91 (4), $546-588$.

Woodford, M., 2002. Imperfect common knowledge and the effects of monetary policy. In: Aghion, P., Frydman, R., Stiglitz, J., Woodford, M. (Eds.), Knowledge, Information, and Expectations in Modern Macroeconomics: In Honor of Edmund S. Phelps. Princeton: Princeton University Press. 


\section{AppendixA. Continuous case}

Let us assume now a continuum of agents in the society. As before each predictor's preferences are generically characterized by:

$$
U\left(e_{i t}, \bar{e}_{t}, \sigma_{e}^{2}, s_{t}\right)
$$

We have verified the existence of a (unique) linear equilibrium strategy expressed by eq. 22 in case of a finite numbers of investors. Here we show that the linear equilibrium is also unique in the continuous case. The best response of investor $-i$ is:

$$
\left.e_{i t}\left(x_{i} ; f ; \rho_{\tilde{y}} ; \rho_{x_{i}}\right)=(1-\delta) \mathbb{E}_{i}\left[s_{t} \mid x_{i t} ; f_{t} ; \rho_{\tilde{y}} ; \rho_{x_{i}}\right]+\delta \mathbb{E}_{i} \int e_{j} d j \mid x_{i t} ; f_{t} ; \rho_{\tilde{y}} ; \rho_{x_{i}}\right]
$$

To make the notation as simple as possible, let us denote $\mathbb{E}_{i}\left[. \mid x_{i t} ; f_{t} ; \rho_{\tilde{y}} ; \rho_{x_{i}}\right]=\mathbb{E}_{i}[$.$] . We can start$ iterating forward such that:

$$
\begin{aligned}
e_{i t} & =(1-\delta) \mathbb{E}_{i}\left[s_{t}\right]+\delta \mathbb{E}_{i}\left[(1-\delta) \int \mathbb{E}_{j}\left[s_{t+1}\right] d j+\delta \int \mathbb{E}_{j}\left[\bar{e}_{t}\right] d j\right] \\
& =(1-\delta) \mathbb{E}_{i}\left[s_{t}\right]+\delta(1-\delta) \mathbb{E}_{i} \overline{\mathbb{E}}\left[s_{t+1}\right]+\delta^{2} \mathbb{E}_{i t} \int \mathbb{E}_{j}\left[\bar{e}_{t}\right] d j \\
& =\vdots \\
& =(1-\delta) \mathbb{E}_{i}\left[s_{t}\right]+\delta(1-\delta) \mathbb{E}_{i} \overline{\mathbb{E}}_{t} s_{t+1}+\delta^{2}(1-\delta) \mathbb{E}_{i} \overline{\mathbb{E}}_{t}^{(2)} s_{t+1}+\ldots
\end{aligned}
$$

$\overline{\mathbb{E}}_{t} s_{t+1}$ is the average expectations operator given by:

$$
\begin{gathered}
\overline{\mathbb{E}}[\cdot] \equiv \int \mathbb{E}_{j}[\cdot] d j \\
\overline{\mathbb{E}}_{t}^{(k)}[\cdot] \equiv \int \mathbb{E}_{j} \overline{\mathbb{E}}^{(k-1)}[\cdot] d j=\overline{\mathbb{E}}_{t} \overline{\mathbb{E}}_{t}^{(k-1)}[\cdot]
\end{gathered}
$$

where $\overline{\mathbb{E}}^{(k)}[\cdot]$ denote the average expectation of order $k$. We get the optimal action of any agent as a simple geometric sum of higher order beliefs:

$$
e_{i t}=(1-\delta) \sum_{k=0}^{\infty} \delta^{k} \mathbb{E}_{i}\left[\overline{\mathbb{E}}_{t}^{(k)} s_{t+1}\right]
$$


Thus higher level of $\delta$ implicitly reveals a greater importance of the weight assigned to the higher order beliefs. To check if the infinite sum is bounded, we need to solve for $\mathbb{E}_{i}\left[\overline{\mathbb{E}}^{(k)} s_{t+1}\right]$. Since the investor-i's expected value of $s_{t}$ is given by eq. 23 , i.e.,

$$
\mathbb{E}_{i t}\left(s_{t+1}\right)=\varphi_{x} x_{i t}+\varphi_{y} \tilde{y}_{t}
$$

while the average expectation across predictors is equal to 24 , i.e.,

$$
\overline{\mathbb{E}}_{t} s_{t+1}=\varphi_{x} \bar{x}_{t}+\varphi_{y} \tilde{y}_{t}
$$

We can rewrite the expectation of $\overline{\mathbb{E}}_{t} s_{t+1}$ for each investor $-i$ as:

$$
\begin{aligned}
\mathbb{E}_{i t} \overline{\mathbb{E}}_{t} s_{t+1} & =\varphi_{x} \mathbb{E}_{i t}\left[\bar{x}_{t+1}\right]+\varphi_{y} \tilde{y}_{t} \\
& =\varphi_{x} \mathbb{E}_{i t}\left[s_{t+1}+\epsilon_{i t+1}\right]+\varphi_{y} \tilde{y}_{t}
\end{aligned}
$$

Then since $\epsilon_{i t+1} \sim N\left(0, \sigma_{\epsilon}^{2}\right)$ and due to symmetry $\left(\bar{s}_{t+1}=s_{t+1}, \forall i\right)$, we easily rewrite:

$$
\begin{aligned}
\mathbb{E}_{i t} \overline{\mathbb{E}}_{t} s_{t+1} & =\varphi_{x} \mathbb{E}_{i t}\left[s_{t+1}\right]+\varphi_{y} \tilde{y}_{t} \\
& =\varphi_{x}\left(\varphi_{x} x_{i t}+\varphi_{y} \tilde{y}_{t}\right)+\varphi_{y} \tilde{y}_{t}= \\
& =\varphi_{x}^{2} x_{i t}+\varphi_{y}^{2} \tilde{y}_{t}
\end{aligned}
$$

this implies that the average expectation of $\overline{\mathbb{E}}_{t} s_{t+1}$ is given by:

$$
\overline{\mathbb{E}}_{t}^{(2)} s_{t+1}=\overline{\mathbb{E}}_{t} \overline{\mathbb{E}}_{t} s_{t+1}=\varphi_{x}^{2} \bar{x}_{t}+\varphi_{y}^{2} \tilde{y}_{t}
$$

Iterating the procedure for any $k$,

$$
\begin{gathered}
\overline{\mathbb{E}}_{t}^{(k)} s_{t+1}=\varphi_{x}^{k} \bar{x}_{t}+\varphi_{y}^{k} \tilde{y}_{t} \\
\mathbb{E}_{i t} \overline{\mathbb{E}}_{t}^{(k)} s_{t+1}=\varphi_{x}^{k+1} x_{i t}+\varphi_{y}^{k+1} \tilde{y}_{t}
\end{gathered}
$$


Now substituting eq. A.4 to eq. A.3, we obtain that:

$$
\begin{aligned}
e_{i t} & =(1-\delta) \sum_{k=0}^{\infty} \delta^{k}\left(\varphi_{x}^{k+1} x_{i t}+\varphi_{y}^{k+1} \tilde{y}_{t}\right) \\
& =\frac{(1-\delta)}{1-\delta \varphi_{x}} \varphi_{x} x_{i t}+\left(1-\frac{(1-\delta)}{1-\delta \varphi_{x}}\right) \varphi_{y} \tilde{y}_{t}
\end{aligned}
$$

while simplifying,

$$
e_{i t}=\frac{(1-\varrho) \rho_{x_{i}}}{(1-\varrho) \rho_{x_{i}}+\rho_{\tilde{y}}} x_{i t}+\frac{\rho_{\tilde{y}}}{(1-\varrho) \rho_{x_{i}}+\rho_{\tilde{y}}} \tilde{y}_{t}
$$

which is exactly the linear equilibrium in a continuum of agents.

\section{AppendixB. MCMC algorithm}

The intuition behind MCMC is to build a Markov chain transition kernel starting from a given initial point and with limiting invariant distribution equal to the posterior distribution of the interested quantities. Under suitable conditions (see Robert and Casella, 1999, chap. 6-7), such a transition kernel converges in distribution to the target posterior density $p(\boldsymbol{\theta} \mid \mathbf{y})$. This Markov chain trajectories are obtained through simulations on the basis of two-steps procedure. First, a new movement is proposed by simulating the new position from a proposal distribution, and second, this move is accepted or rejected according to some suitable probabilities that depend on the likelihood function and on the prior distribution of the parameters $p(\boldsymbol{\theta})$. In a nutshell, given a starting value for the parameter's vector $\boldsymbol{\theta}^{(0)}$, we simulate trajectories of the Markov chain $\left\{\boldsymbol{\theta}^{(j)}, j=1, \ldots, n\right\}$ whose draws converge to the posterior distribution. Once convergence is achieved, inference can be based on the generated serially dependent sample simulated from the posterior. More precisely, estimates of the posterior means $\mathrm{E}_{p(\boldsymbol{\theta} \mid \mathbf{y})}[\boldsymbol{\theta}]$ are obtained by averaging over the realization of the chains, i.e., $\hat{\boldsymbol{\theta}}=n^{-1} \sum_{j=1}^{n} \boldsymbol{\theta}^{(j)}$. To account for serial correlation induced by the Markovian nature of this procedure, we estimate the numerical standard error of the sample posterior mean using the approach implemented, for instance, by Kim et al. (1998). In this application, $\boldsymbol{\theta}$ are the structural parameters of the model including, amongst others $\lambda, \delta, \rho_{x_{i}}, \rho_{f}$ and $\rho_{s}$.

In the MCMC literature, there are many different ways to make a step from the Markov chain. Our inferential procedure is based on a Random Walk Metropolis-Hastings algorithm, that has been proved to be effective in the DSGE framework (An and Schorfheide, 2007), and in which the proposal distribution 
depends uniquely on the current state of the chain at time $j$, i.e., $q\left(\boldsymbol{\theta} \mid \boldsymbol{\theta}^{(j)}\right)$. Once parameters are updated, the exact likelihood function is evaluated through the Kalman filter to eqs. (37) - 39)

We thus propose through the random-walk step new values for these parameters and then we compute the reduced form to evaluate the exact likelihood.

The procedure can be summarized as follows

\section{MCMC algorithm}

- Initialize the chain at $\boldsymbol{\theta}^{(0)}$

- At step $j=1, \ldots, n$

- Update $\boldsymbol{\theta}$ in block through a random walk Metropolis-Hastings scheme

$$
\boldsymbol{\theta}^{*} \sim q\left(\boldsymbol{\theta} \mid \boldsymbol{\theta}^{(j-1)}\right)
$$

- Compute $L\left(\mathbf{y} \mid \boldsymbol{\theta}^{*}\right)$ through Kalman filter;

- Compute the acceptance probability $\alpha\left(\boldsymbol{\theta}^{(j-1)}, \boldsymbol{\theta}^{*}\right)$ defined as

$$
\alpha\left(\boldsymbol{\theta}^{(j-1)}, \boldsymbol{\theta}^{*}\right)=\frac{p\left(\boldsymbol{\theta}^{*}\right) L\left(\mathbf{y} \mid \boldsymbol{\theta}^{*}\right) q\left(\boldsymbol{\theta}^{(j-1)} \mid \boldsymbol{\theta}^{*}\right)}{p\left(\boldsymbol{\theta}^{(j-1)}\right) L\left(\mathbf{y} \mid \boldsymbol{\theta}^{(j-1)}\right) q\left(\boldsymbol{\theta}^{*} \mid \boldsymbol{\theta}^{(j-1)}\right)}
$$

- Draw $u$ from an $U(0,1)$ random variable. If $\alpha\left(\boldsymbol{\theta}^{(j-1)}, \boldsymbol{\theta}^{*}\right) \leq u$

* Then $\boldsymbol{\theta}^{(j)}=\boldsymbol{\theta}^{*}$;

* Else $\boldsymbol{\theta}^{(j)}=\boldsymbol{\theta}^{(j-1)}$;

- $j=j+1$

\section{AppendixC. Forecast Error Variance Decomposition for $\Delta x_{t}$}

To obtain the FEVD for the observed data, we recur to the following MA representation of the economic system. The reduced form model is

$$
\boldsymbol{x}_{t}=\Theta_{x} \boldsymbol{x}_{t-1}+\epsilon_{t}, \quad \mathrm{E}\left[\epsilon_{t} \epsilon_{t}^{\prime}\right]=\Theta_{\epsilon} \Theta_{\epsilon}^{\prime}
$$


or, using a polynomial notation with lag operator $L$,

$$
\Theta_{x}(L) \boldsymbol{x}_{t}=\epsilon_{t}
$$

In particular some of the processes involved are random walks. In the following, $n$ is the dimension of $\boldsymbol{x}_{t}$ whereas $n-r$ is the number of random walks.

By looking at the rank of $\Theta_{x}$, we observe that for all the posterior parameters from the MCMC algorithm, there exist $r$ cointegration relationships, then an MA representation of $\Delta \boldsymbol{x}_{t}$ exists. Consider that,

$$
\Theta_{x}(L)=\left[\begin{array}{cc}
(1-L) I_{n-r} & 0_{n-r, r} \\
-A_{r, n-r} L & I-L B_{r, r}
\end{array}\right]
$$

Following Engle and Yoo (1991), the autoregressive polynomial can be factorized as $\Theta_{x}(L)=U(L) M(L) V(L)$, to disentangle the stationary from the non-stationary part of the model. In particular we get

$$
\Theta_{x}(L)=\left[\begin{array}{cc}
I_{n-r} & 0_{n-r, r} \\
0_{r, n-r} & I_{r, r}
\end{array}\right]\left[\begin{array}{cc}
(1-L) I_{n-r} & 0_{n-r, r} \\
0_{r, n-r} & I_{r, r}
\end{array}\right]\left[\begin{array}{cc}
I_{n-r} & 0_{n-r, r} \\
-A_{r, n-r} L & I-L B_{r, r}
\end{array}\right]
$$

and the corresponding matrix

$$
V=\left[\begin{array}{cc}
I_{n-r} & 0_{n-r, r} \\
A_{r, n-r} L & B_{r, r}
\end{array}\right]
$$

Some tedious algebra allows to get

$$
\Delta \boldsymbol{x}_{t}=\left[\begin{array}{cc}
I_{n-r} & 0_{n-r, r} \\
\sum_{i=1}^{\infty} \Theta_{21}^{(i)} L^{i} & \sum_{i=1}^{\infty} \Theta_{22}^{(i)} L^{i}(1-L)
\end{array}\right] \epsilon_{t}
$$

in which $\Theta_{21}^{(i)}$ and $\Theta_{22}^{(i)}$ are respectively the lower left and right blocks of $V^{i}$.

\section{AppendixD. Foreign exchange Consensus Survey data}

On the second Monday of every month, Foreign Exchange Consensus Forecasts (FECF) asks their panelists to forecast spot rates for the use against the euro over a range of time horizons. Each panelist provides a series of forecasts with different maturities: one that refers to the next month, one to the next 
quarter, one to the next year and one to the next two years. Panelists are almost 250, and around 40 per each publication on average are identified individually with their names. Nonetheless, FECF reports in the publication the Consensus forecast which is the mean of all forecasts received by the company, including those panelists whose names are not explicitly indicated. The forecasts of the panelists are polled into an average forecast that is reported as well, and is defined in the publication as Other Forecasters. Let us refer to the individual forecasts as the forecasts of the components of the panel whose identity is explicitly indicated in the publication 26

The companies in the poll are indicated with their names in the publication and listed in descending order of their 1 year percentage change estimates. Consequently, the order in which the panelists appear in the list may change every month. The composition of the panelists whose names appear explicitly is not always the same. There are institutions that provide predictions in almost every publication in the time span considered, while some others provide a lower frequency. There are also cases in which the company is present in the list but the forecast is not provided. This is indicated with the label $n a$ in the value for the forecast 27 . The names of the companies included in our database are presented in Table D.4,

Forex trading shows a very fast modernization in the last years fundamentally based on electronic trading leading to a complete restructuring of forex market. Large banks have the necessary resources to develop sophisticated proprietary trading platforms through which satisfy their own customers. They also provide trading service to smaller banks, which have mostly withdrawn from the market due to the high costs associated with the investment in these platforms. Small banks have nonetheless maintained their ability to provide liquidity to their customers using the proprietary platforms of the large banks but under their names. This procedure is called white labeling and is certainly efficient for the market functioning, although it has driven a concentration of market information. In this case large banks can observe directly small banks' trading flows and extract from these data possibly relevant information. The concentration driven by the phenomenon of white labeling is striking since the three larger bank trading platforms account for $70 \%$ of the total market share 28

\footnotetext{
${ }^{26}$ We refer to Consensus forecasts and Other forecasters indicating the same group of forecasters in the publication as previously described.

${ }^{27}$ When we created our database we considered both the lack of a prediction of the company for that month and of the presence of the company in the list as $N A$

${ }^{28}$ See James et al. (2012), page 30, about the Euromoney FX Survey, where the list of all the institutions with a trading platform is reported.
} 
Table D.4: Predictions of individual forecasters: missing observations in absolute value and percentage on the total number of observations in the sample. Last column: companies with proprietary trading platforms (Euromoney FX survey).

\begin{tabular}{lccc}
\hline & $\mathrm{n}$ missing & \% on total & white label \\
\hline Bank.of.Tokio.Mitsubushi & 1 & 1 & \\
Barclays.Capital & 31 & 40 & yes \\
BNP.Paribas & 7 & 9 & \\
BoA.Merril.Linch & 4 & 5 & \\
Citygroup & 47 & 60 & yes \\
Commerzbank & 4 & 5 & \\
Deutsche.Bank.Research & 18 & 23 & yes \\
General.Motors & 0 & 0 & \\
HSBC & 1 & 1 & yes \\
IHS.Global.Insight & 0 & 0 & \\
JPMorgan & 10 & 13 & yes \\
Oxford.Economics & 7 & 9 & \\
Royal.Bank.of.Canada & 0 & 0 & \\
UBS & 3 & 4 & yes \\
WestLB & 3 & 4 & \\
\hline
\end{tabular}

Other variables are reported in the same panel where forecasts are indicated, i.e., the annual percentage change of the consensus rate and the discount (or premium) of the survey consensus forecast with respect to the spot rate at the date of the forecast. In the same page, the FECF presents data on Current Account Balances in usd and consensus forecast on current account balances for major countries of the euro area ${ }^{29}$ Country risk indicators are also reported for information to the subscribers: current account and budget balance (in \% of GDP) for the current year and the prediction for the next year; public debt of the preceding year (in \% of GDP) and sovereign debt ratings of both Moody's and S\&P. Two figures are presented to the readers: in the first, the usd per euro exchange rate together with usd less euro 3 Month interest rate futures differential contracts(as of the end of the following quarter). In the second, the Yen/euro cross rate to explore currency linkages with the japanese currency. The yen per usd exchange rate is the only other currency for which the prediction of the panelists appears in detail in the publication every month.

\section{AppendixD.1. Data availability and database construction}

We study monthly forecasts of the usd against the euro exchange rate for a period ranging from January 2006 to June 2012, which amounts to 78 monthly predictions over this time span. As previously

\footnotetext{
${ }^{29}$ In detail: Germany, France, Italy, Austria, Belgium, Finland, Greece, Ireland, Netherlands, Portugal, Spain, and Euro zone.
} 
mentioned, the number and the identity of forecasters is not constant so we had to build a database using the information of the individual forecast in a constructive way. We implemented a conservative approach. We collected the predictions of the institutions that appeared at least once among the individual forecasts in the time span considered. Then, we collected all available forecasts and recorded a missing value the occurrence when the predictor was either non present in the panel for that month publication or when the company prediction for the month was indicated as 'na'. Consequently we obtained individual predictions for 15 institutions:

\section{AppendixD.2. Data on fundamentals}

The data source for all macro data is Datastream.

\section{Spot exchange rate data:}

We collected data to euro exchange rate at daily frequency from december 2005 to august 2012. To obtain a monthly series for the exchange rate we implemented the choices of Fratzscher et al. (2012). We use nominal bilateral exchange rate changes vis-a-vis the reference currency, in the benchmark specification using changes over the past month. Since we know the exact day when the surveys were conducted, these exchange rate changes are calculated relative to the exchange rate of the previous business day.

GDP: data on GDP are at quarterly frequency. We need to disaggregate them in order to have a monthly frequency that matches the survey frequency. To obtain the monthly data we use the following macroeconomic indicators following Golinelli and Parigi: Long term interest rate (per cent per annum), harmonized unemployment rate; Retail trade; Industrial production. For the money supply the difference of the log of M2 for US $\$$ and euro (frequency). 


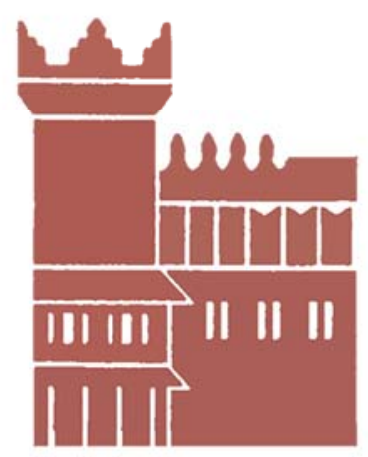

Alma Mater Studiorum - Università di Bologna DEPARTMENT OF ECONOMICS

Strada Maggiore 45

40125 Bologna - Italy

Tel. +39051 2092604

Fax +390512092664

http://www.dse.unibo.it 